\title{
Tribute to \\ Vladimir Arnold
}

\author{
Boris Khesin and Serge Tabachnikov, \\ Coordinating Editors
}

Vladimir Arnold, an eminent mathematician of our time, passed away on June 3, 2010, nine days before his seventy-third birthday. This article, along with one in the next issue of the Notices, touches on his outstanding personality and his great contribution to mathematics.

A word about spelling: we use "Arnold", as opposed to "Arnol'd"; the latter is closer to the Russian pronunciation, but Vladimir Arnold preferred the former (it is used in numerous translations of his books into English), and we use it throughout.

\section{Arnold in His Own Words}

In 1990 the second author interviewed V. Arnold for a Russian magazine Kvant (Quantum). The readership of this monthly magazine for physics and mathematics consisted mostly of high school students, high school teachers, and undergraduate students; the magazine had a circulation of about 200,000. As far as we know, the interview was never translated into English. We translate excerpts from this interview; ${ }^{1}$ the footnotes are ours.

Q: How did you become a mathematician? What was the role played by your family, school, mathematical circles, Olympiads? Please tell us about your teachers.

A: I always hated learning by rote. For that reason, my elementary school teacher told my parents that a moron, like myself, would never manage to master the multiplication table.

Boris Khesin is professor of mathematics at the University of Toronto. His email address is khes in@math. toronto. edu.

Serge Tabachnikov is professor of mathematics at Pennsylvania State University. His email address is tabachni@ math. psu.edu.

${ }^{1}$ Full text is available in Russian on the website of Kvant magazine (July 1990), http://kvant . mi rror1. mccme. $\mathrm{ru} /$.

DOI: http://dx.doi.org/10.1090/noti810
My first mathematical revelation was when I met my first real teacher of mathematics, Ivan Vassilievich Morozkin. I remember the problem about two old ladies who started simultaneously from two towns to-

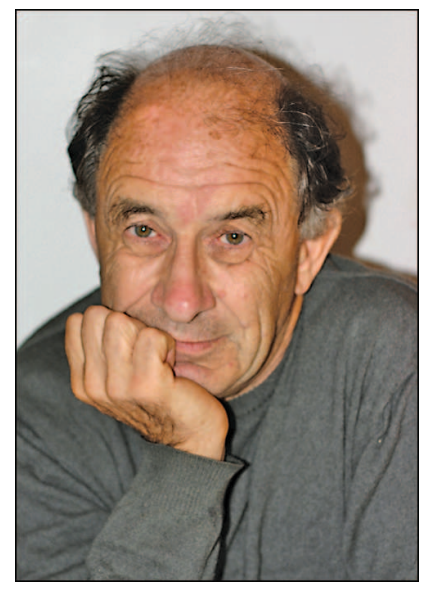
ward each other, met at noon, and who reached the opposite towns at 4 p.m. and 9 p.m., respectively. The question was when they started their trip.

We didn't have algebra yet. I invented an "arithmetic" solution (based on a scaling-or similarityargument) and experienced a joy of discovery; the desire to Vladimir Igorevich Arnold experience this joy again was what made me a mathematician. A. A. Lyapunov organized at his home "Children Learned Society". The curriculum included mathematics and physics, along with chemistry and biology, including genetics that was just recently banned ${ }^{2}$ (a son of one of our best geneticists was my classmate; in a questionnaire, he wrote: "my mother is a stay-at-home mom; my father is a stay-at-home dad").

Q: You have been actively working in mathematics for over thirty years. Has the attitude of society towards mathematics and mathematicians changed?

A: The attitude of society (not only in the USSR) to fundamental science in general, and to mathematics in particular, is well described by I. A. Krylov

\footnotetext{
${ }^{2}$ In 1948 genetics was officially declared "a bourgeois pseudoscience" in the former Soviet Union.
} 


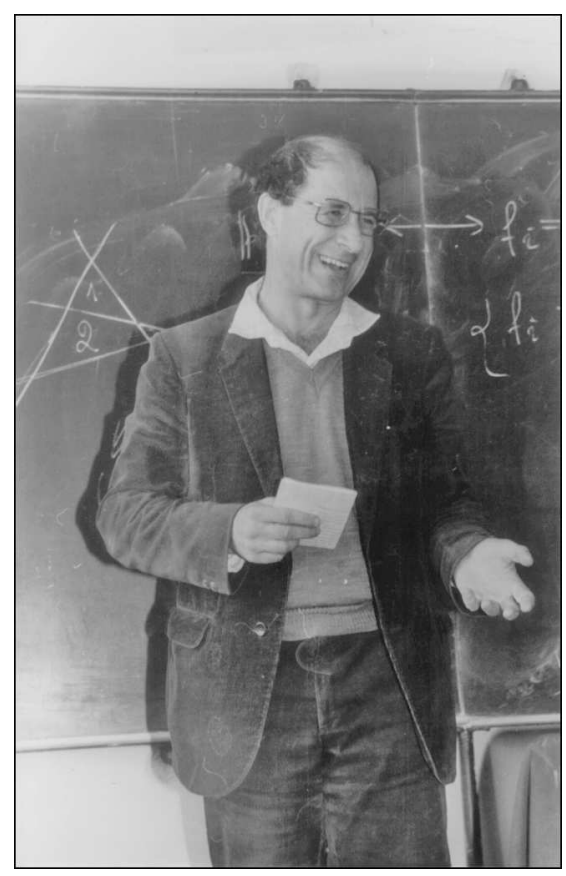

Vladimir Arnold, circa 1985.

in the fable "The hog under the oak". ${ }^{3}$ In the 1930s and 1940s, mathematics suffered in this country less than other sciences. It is well known that Viète was a cryptographer in the service of Henry IV of France. Since then, certain areas of mathematics have been supported by all governments, and even Beria $^{4}$ cared about preservation of mathematical culture in this country.

In the last thirty years the prestige of mathematics has declined in all countries. I think that mathematicians are partially to be blamed as well (foremost, Hilbert and Bourbaki), particularly the ones who proclaimed that the goal of their science was investigation of all corollaries of arbitrary systems of axioms.

Q: Does the concept of fashion apply to mathematics?

A: Development of mathematics resembles a fast revolution of a wheel: sprinkles of water are flying in all directions. Fashion-it is the stream that leaves the main trajectory in the tangential direction. These streams of epigone works attract the most attention, and they constitute the main mass, but they inevitably disappear after a while because they parted with the wheel. To remain on the wheel, one must apply the effort in the direction perpendicular to the main stream.

A mathematician finds it hard to agree that the introduction of a new term not supported by

\footnotetext{
${ }^{3}$ See A. Givental and E. Wilson-Egolf's (slightly modern ized) translation of this early nineteenth-century Russian fable at the end of this interview.

${ }^{4}$ The monstrous chief of Stalin's secret police.
}

new theorems constitutes substantial progress. However, the success of "cybernetics", "fractals", "synergetics", "catastrophe theory", and "strange attractors" illustrates the fruitfulness of word creation as a scientific method.

Q: Mathematics is a very old and important part of human culture. What is your opinion about the place of mathematics in cultural heritage?

A: The word "mathematics" means science about truth. It seems to me that modern science (i.e., theoretical physics along with mathematics) is a new religion, a cult of truth, founded by Newton three hundred years ago.

Q: When you prove a theorem, do you "create" or "discover" it?

A: I certainly have a feeling that I am discovering something that existed before me.

Q: You spend much time popularizing mathematics. What is your opinion about popularization? Please name merits and demerits of this hard genre.

A: One of the very first popularizers, M. Faraday, arrived at the conclusion that "Lectures which really teach will never be popular; lectures which are popular will never teach." This Faraday effect is easy to explain: according to N. Bohr, clearness and truth are in a quantum complementarity relation.

Q: Many readers of Kvant aspire to become mathematicians. Are there "indications" and "contraindications" to becoming a mathematician, or can anyone interested in the subject become one? Is it necessary for a mathematician-to-be to successfully participate in mathematical Olympiads?

A: When 90-year-old Hadamard was telling A. N. Kolmogorov about his participation in Concours Général (roughly corresponding to our Olympiads), he was still very excited: Hadamard won only the second prize, while the student who had won the first prize also became a mathematician, but a much weaker one!

Some Olympiad winners later achieve nothing, and many outstanding mathematicians had no success in Olympiads at all.

Mathematicians differ dramatically by their time scale: some are very good tackling 15-minute problems, some are good with the problems that require an hour, a day, a week, the problems that take a month, a year, decades of thinking. A. N. Kolmogorov considered his "ceiling" to be two weeks of concentrated thinking.

Success in an Olympiad largely depends on one's sprinter qualities, whereas serious mathematical research requires long distance endurance (B. N. Delaunay used to say, "A good theorem takes not 5 hours, as in an Olympiad, but 5,000 hours").

There are contraindications to becoming a research mathematician. The main one is lack of love of mathematics. 


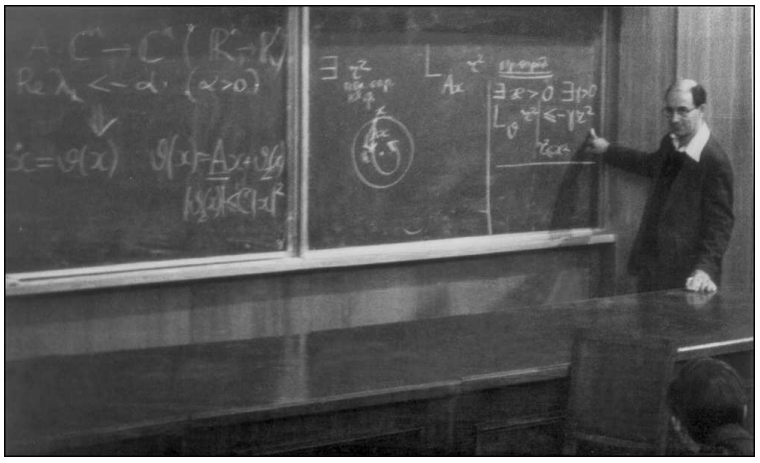

Teaching at Moscow State University, 1983.

But mathematical talents can be very diverse: geometrical and intuitive, algebraic and computational, logical and deductive, natural scientific and inductive. And all kinds are useful. It seems to me that one's difficulties with the multiplication table or a formal definition of half-plane should not obstruct one's way to mathematics. An extremely important condition for serious mathematical research is good health.

$\mathrm{Q}$ : Tell us about the role of sport in your life.

A: When a problem resists a solution, I jump on my cross-country skis. After forty kilometers a solution (or at least an idea for a solution) always comes. Under scrutiny, an error is often found. But this is a new difficulty that is overcome in the same way.

\section{The Hog Under the Oak}

A Hog under a mighty Oak

Had glutted tons of tasty acorns, then, supine, Napped in its shade; but when awoke,

He, with persistence and the snoot of real swine,

The giant's roots began to undermine.

"The tree is hurt when they're exposed."

A Raven on a branch arose.

"It may dry up and perish-don't you care?"

"Not in the least!" The Hog raised up its head.

"Why would the prospect make me scared?

The tree is useless; be it dead

Two hundred fifty years, I won't regret a second.

Nutritious acorns-only that's what's reckoned!"-

"Ungrateful pig!" the tree exclaimed with scorn.

"Had you been fit to turn your mug around

You'd have a chance to figure out

Where your beloved fruit is born."

Likewise, an ignoramus in defiance

Is scolding scientists and science,

And all preprints at lanl_dot_gov,

Oblivious of his partaking fruit thereof.

\section{Arnold's Doctoral Students}

The list below includes those who defended their Ph.D. theses under Arnold's guidance. We have to admit that it was difficult to compile. Along with straightforward cases when Arnold supervised the thesis and was listed as the person's Ph.D. advisor, there were many other situations. For example, in Moscow State University before perestroika, a Ph.D. advisor for a foreigner had to be a member of the Communist Party, so in such cases there was a different nominal Ph.D. advisor while Arnold was supervising the student's work. In other cases there were two co-advisors or there was a different advisor of the Ph.D. thesis, while the person defended the Doctor of Science degree (the second scientific degree in Russia) under Arnold's supervision. In these "difficult cases" the inclusion in the list below is based on "self-definition" as an Arnold student rather than on a formality. We tried to make the list as complete and precise as possible, but we apologize in advance for possible omissions: there were many more people whose work Arnold influenced greatly and who might feel they belong to Arnold's school.

Names are listed chronologically according to the defense years, which are given in parentheses. Many former Arnold's students defended the second degree, the Doctor of Science or Habilitation, but we marked it only in the cases where the first degree was not under Arnold's supervision.

Edward G. Belaga (1965)

Andrei M. Leontovich (1967)

Yulij S. Ilyashenko (1969) (1994, DSci)

Anatoly G. Kushnirenko (1970)

Askold G. Khovanskii (1973)

Nikolai N. Nekhoroshev (1973)

Alexander S. Pyartli (1974)

Alexander N. Varchenko (1974)

Sabir M. Gusein-Zade (1975)

Alexander N. Shoshitaishvili (1975)

Rifkat I. Bogdanov (1976)

Lyudmila N. Bryzgalova (1977)

Vladimir M. Zakalyukin (1977)

Emil Horozov (1978)

Oleg V. Lyashko (1980)

Olga A. Platonova (1981)

Victor V. Goryunov (1982)

Vladimir N. Karpushkin (1982)

Vyacheslav D. Sedykh (1982)

Victor A. Vassiliev (1982)

Aleksey A. Davydov (1983)

Elena E. Landis (1983)

Vadim I. Matov (1983)

Sergei K. Lando (1986)

Inna G. Scherbak (1986)

Oleg P. Scherbak (1986)

Victor I. Bakhtin (1987)

Alexander B. Givental (1987)

Mikhail B. Sevryuk (1988)

Anatoly I. Neishtadt (1976) (1989, DSci)

Ilya A. Bogaevsky (1990)

Boris A. Khesin (1990)

Vladimir P. Kostov (1990) 
Boris Z. Shapiro (1990)

Maxim E. Kazarian (1991)

Ernesto Rosales-Gonzalez (1991)

Oleg G. Galkin (1992)

Michael Z. Shapiro (1992)

Alexander Kh. Rakhimov (1995)

Francesca Aicardi (1996)

Yuri V. Chekanov (1997)

Emmanuel Ferrand (1997)

Petr E. Pushkar (1998)

Jacques-Olivier Moussafir (2000)

Mauricio Garay (2001)

Fabien Napolitano (2001)

Ricardo Uribe-Vargas (2001)

Mikhail B. Mishustin (2002)

Adriana Ortiz-Rodriguez (2002)

Gianmarco Capitanio (2004)

Oleg N. Karpenkov (2005)

Alexander M. Lukatsky (1975) (2006, DSci)

\section{Alexander Givental}

\section{To Whom It May Concern}

$$
\begin{array}{r}
\text { Но есть и Божий суд ... } \\
\text { М. Ю. Лермонтов, "Смерть Поэта"6 }
\end{array}
$$

Posthumous memoirs seem to have the unintended effect of reducing the person's life to a collection of stories. For most of us it would probably be a just and welcome outcome, but for Vladimir Arnold, I think, it would not. He tried and managed to tell us many different things about mathematics, education, and beyond, and in many cases we've been rather slow listening or thinking, so I believe we will be returning again and again not only to our memories of him but to his own words as well. What is found below is not a memoir, but a recommendation letter, albeit a weak one, for he did not get the prize, and yet hopefully useful as an interim attempt to overview his mathematical heritage.

January 25, 2005

Dear Members of [the name of the committee],

You have requested my commentaries on the work of Vladimir Arnold. Writing them is an honorable and pleasurable task for me.

In the essence the task is easy:

Yes, Vladimir Arnold fully merits [the name of the prize] since his achievements are of extraordinary depth and influence.

His work indeed resolves fundamental problems, and introduces unifying principles, and opens up major new areas, and (at least in some of

Alexander Givental is professor of mathematics at the University of California, Berkeley. His email address is giventh@math. berkeley. edu.

${ }^{6}$ Yet, there is God's Court, too..., M. Yu. Lermontov, "Death of Poet".

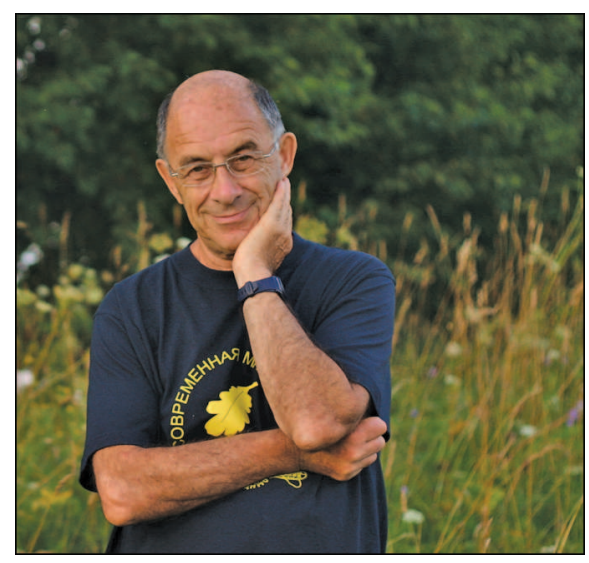

At Dubna, 2006.

these areas) it introduces powerful new techniques too.

On the other hand, writing this letter is not easy, mainly because the ways Arnold's work contributes to our knowledge are numerous and go far beyond my personal comprehension. As Arnold's student, I am quite familiar with those aspects of his work which inspired my own research. Outside these areas, hopefully, I will be able to convey the conventional wisdom about Arnold's most famous achievements. Yet this leaves out the ocean of numerous, possibly less famous but extremely influential, contributions, of which I have only partial knowledge and understanding. So, I will have to be selective here and will mention just a handful of examples which I am better familiar with and which for this reason may look chosen randomly.

Perhaps the most legendary, so to speak, of Arnold's contributions is his work on small denominators, ${ }^{7}$ followed by the discovery of Arnold's diffusion, ${ }^{8}$ and known now as part of the Kolmogorov-Arnold-Moser theory. Among other things, this work contains an explanation (or, depending on the attitude, a proof, and a highly technical one) of stability of the solar planetary system. Even more importantly, the KAM theory provides a very deep insight into the real-world dynamics (perhaps one of the few such insights so far, one more being stability of Anosov's systems) and

\footnotetext{
${ }^{7}$ Small denominators III. Problems of stability of motion in classical and celestial mechanics, Uspekhi Mat. Nauk 18 (1963), no. 6, 91-192, following Small denominators I. Mappings of a circle onto itself, Izvestia AN SSSR, Ser. Mat. 25 (1961), 21-86, Small denominators II. Proof of a theorem of A. N. Kolmogorov on the preservation of conditionally periodic motions under a small perturbation of the Hamiltonian, Uspekhi Mat. Nauk 18 (1963), no. 5, 13-40, and a series of announcements in DAN SSSR.

${ }^{8}$ Instability of dynamical systems with many degrees of freedom, DAN SSSR 156 (1964), 9-12.
} 
is widely regarded as one of the major discoveries of twentieth-century mathematical physics.

Symplectic geometry has established itself as a universal geometric language of Hamiltonian mechanics, calculus of variations, quantization, representation theory and microlocal analysis of differential equations. One of the first mathematicians who understood the unifying nature of symplectic geometry was Vladimir Arnold, and his work played a key role in establishing this status of symplectic geometry. In particular, his monograph Mathematical Methods of Classical Mechanics ${ }^{9}$ has become a standard textbook, but thirty years ago it indicated a paradigm shift in a favorite subject of physicists and engineers. The traditional "analytical" or "theoretical" mechanics got suddenly transformed into an active region of modern mathematics populated with Riemannian metrics, Lie algebras, differential forms, fundamental groups, and symplectic manifolds.

Just as much as symplectic geometry is merely a language, symplectic topology is a profound problem. Many of the best results of such powerful mathematicians as Conley, Zehnder, Gromov, Floer, Hofer, Eliashberg, Polterovich, McDuff, Salamon, Fukaya, Seidel, and a number of others belong to this area. It would not be too much of an overstatement to say that symplectic topology has developed from attempts to solve a single problem: to prove the Arnold conjecture about Hamiltonian fixed points and Lagrangian intersections. ${ }^{10}$ While the conjecture has been essentially proved $^{11}$ and many new problems and ramifications discovered, the theory in a sense continues to explore various facets of that same topological rigidity property of phase spaces of Hamiltonian mechanics that goes back to Poincaré and Birkhoff and whose symplectic nature was first recognized by Arnold in his 1965 notes in Comptes Rendus.

Arnold's work in Riemannian geometry of infinite-dimensional Lie groups had almost as much of a revolutionizing effect on hydrodynamics as his work in small denominators produced in classical mechanics. In particular, Arnold's seminal

\footnotetext{
${ }^{9}$ Nauka, Moscow, 1974.

${ }^{10}$ First stated in Sur une propriété topologique des applications globalement canoniques de la mécanique classique, C. R. Acad. Sci. Paris 261 (1965), 3719-3722, and reiterated in a few places, including an appendix to Math Methods....

${ }^{11}$ By Hofer (1986) for Lagrangian intersections and by Fukaya-Ono (1996) for Hamiltonian diffeomorphisms, while "essentially" refers to the fact that the conjectures the way Arnold phrased them in terms of critical point of functions rather than (co)homology, and especially in the case of possibly degenerate fixed or intersection points, still remain open (and correct just as likely as not, but with no counterexamples in view).
}

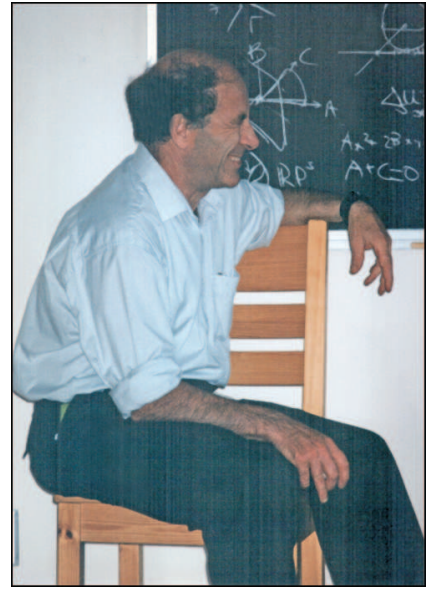

Between the lectures at Arnoldfest, 1997.

paper in Annales de L'Institut Fourier ${ }^{12}$ draws on his observation that flows of incompressible fluids can be interpreted as geodesics of right-invariant metrics on the groups of volume-preserving diffeomorphisms. Technically speaking, the aim of the paper is to show that most of the sectional curvatures of the area-preserving diffeomorphism group of the standard 2-torus are negative and thus the geodesics on the group typically diverge exponentially. From time to time this result makes the news as a "mathematical proof of impossibility of long-term weather forecasts". More importantly, the work had set Euler's equations on coadjoint orbits as a blueprint and redirected the attention in many models of continuum mechanics toward symmetries, conservation laws, relative equilibria, symplectic reduction, topological methods (in works of Marsden, Ratiu, Weinstein, Moffat, and Freedman among many others). ${ }^{13}$

Due to the ideas of Thom and Pham and fundamental results of Mather and Malgrange, singularity theory became one of the most active fields of the seventies and eighties, apparently with two leading centers: Brieskorn's seminar in Bonn and Arnold's seminar in Moscow. The theory of critical points of functions and its applications to classification of singularities of caustics, wave fronts and short-wave asymptotics in geometrical optics as well as their relationship with the ADE-classification are perhaps the most famous (among uncountably many other) results

\footnotetext{
${ }^{12}$ Sur la géométrie différentielle des groupes de Lie de dimension infinie et ses applications à l'hydrodynamique des fluides parfaits, Ann. Inst. Fourier 16:1 (1966), 319361 , based on a series of earlier announcements in $C$. $R$. Acad. Sci. Paris.

${ }^{13}$ As summarized in the monograph Topological Methods in Hydrodynamics, Springer-Verlag, 1998, by Arnold and Khesin.
} 


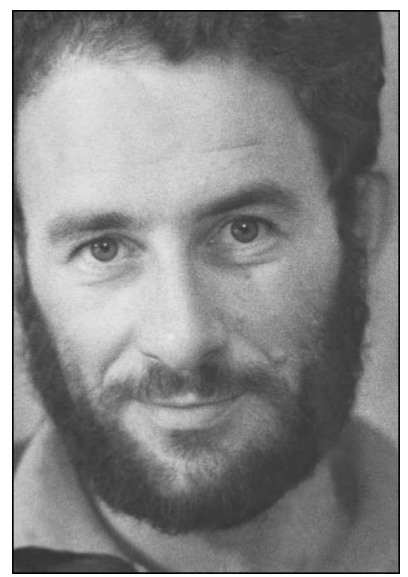

V. Arnold, 1968.

of Arnold in singularity theory. ${ }^{14}$ Arnold's role in this area went, however, far beyond his own papers.

Imagine a seminar of about thirty participants: undergraduates writing their first research papers, graduate students working on their dissertation problems, postgraduates employed elsewhere as software engineers but unwilling to give up their dream of pursuing mathematics even if only as a hobby, several experts-Fuchs, Dolgachev, Gabrielov, Gusein-Zade, Khovansky, Kushnirenko, Tyurin, Varchenko, Vassiliev-and the leader, Arnold-beginning each semester by formulating a bunch of new problems, giving talks or listening to talks, generating and generously sharing new ideas and conjectures, editing his students' papers, and ultimately remaining the only person in his seminar who would keep in mind everyone else's works-in-progress and understand their relationships. Obviously, a lion's share of his students' achievements (and among the quite famous ones are the theory of Newton polyhedra by Khovansky and Kushnirenko or Varchenko's results on asymptotical mixed Hodge structures and semicontinuity of Steenbrink spectra) is due to his help, typically in the form of working conjectures, but every so often through his direct participation (for, with the exception of surveys and obituaries, Arnold would refuse to publish joint papers-we will learn later why).

Moreover, under Arnold's influence, the elite branch of topology and algebraic geometry studying singular real and complex hypersurfaces was transformed into a powerful tool of applied

\footnotetext{
${ }^{14}$ Normal forms of functions near degenerate critical points, the Weyl groups $A_{k}, D_{k}, E_{k}$ and Lagrangian singularities, Funct. Anal. Appl. 6, no. 4 (1972), 3-25; see also Arnold's inspiring paper in Proceedings of the ICM-74 Vancouver and the textbooks Singularities of Differential Maps, Vols. I and II, by Arnold, Gusein-Zade and Varchenko.
}

mathematics dealing with degenerations of all kinds of mathematical objects (metamorphoses of wave fronts and caustics, evolutes, evolvents and envelopes of plane curves, phase diagrams in thermodynamics and convex hulls, accessibility regions in control theory, differential forms and Pfaff equations, symplectic and contact structures, solutions of Hamilton-Jacobi equations, the Hamilton-Jacobi equations themselves, the boundaries between various domains in functional spaces of all such equations, etc.) and merging with the theory of bifurcations (of equilibria, limit cycles, or more complicated attractors in ODEs and dynamical systems). Arnold had developed a unique intuition and expertise in the subject, so that when physicists and engineers would come to him asking what kind of catastrophes they should expect in their favorite problems, he would be able to guess the answers in small dimensions right on the spot. In this regard, the situation would resemble experimental physics or chemistry, where personal expertise is often more important than formally registered knowledge.

Having described several (frankly, quite obvious) broad areas of mathematics reshaped by Arnold's seminal contributions, I would like to turn now to some more specific classical problems which attracted his attention over a long time span.

The affirmative solution of the 13th Hilbert problem (understood as a question about superpositions of continuous functions) given by Arnold in his early (essentially undergraduate) work ${ }^{15}$ was the beginning of his interest in the "genuine" (and still open) Hilbert's problem: Can the root of the general degree 7 polynomial considered as an algebraic function of its coefficients be written as a superposition of algebraic functions of 2 variables? The negative ${ }^{16}$ solution to the more general question about polynomials of degree $n$ was given by Arnold in 1970 for $n=2^{r} .{ }^{17}$ The result was generalized by V. Lin. Furthermore, Arnold's approach, based on his previous study of cohomology of braid groups, later gave rise to Smale's concept of topological complexity of algorithms and Vassiliev's results on this subject. Even more importantly, Arnold's study of braid groups via topology of configuration spaces ${ }^{18}$ was generalized by Brieskorn to E. Artin's braid groups associated with reflection groups. The latter inspired OrlikSolomon's theory of hyperplane arrangements,

\footnotetext{
${ }^{15}$ On the representations of functions of several variables as a superposition of functions of a smaller number of variables, Mat. Prosveshchenie (1958), 41-46.

${ }^{16}$ That is, positive in Hilbert's sense.

${ }^{17}$ Topological invariants of algebraic functions. II, Funct. Anal. Appl. 4 (1970), no. 2, 1-9.

${ }^{18}$ The cohomology ring of the group of dyed braids, Mat. Zametki 5 (1969), 227-231.
} 
K. Saito-Terao's study of free divisors, Gelfand's approach to hypergeometric functions, Aomoto's work on Yang-Baxter equations, and VarchenkoSchekhtman's hypergeometric "Bethe ansatz" for solutions of Knizhnik-Zamolodchikov equations in conformal field theory.

Arnold's result ${ }^{19}$ on the 16th Hilbert problem, Part I, about disposition of ovals of real plane algebraic curves, was immediately improved by Rokhlin (who applied Arnold's method but used more powerful tools from the topology of 4-manifolds). This led Rokhlin to his proof of a famous conjecture of Gudkov (who corrected Hilbert's expectations in the problem), inspired many new developments (due to Viro and Kharlamov among others), and is considered a crucial breakthrough in the history of real algebraic geometry.

Among other things, the paper of Arnold outlines an explicit diffeomorphism between $S^{4}$ and the quotient of $C P^{2}$ by complex conjugation..$^{20}$ The fact was rediscovered by Kuiper in 1974 and is known as Kuiper's theorem [31]. Arnold's argument, based on hyperbolicity of the discriminant in the space of Hermitian forms, was recently revived in a far-reaching paper by $\mathrm{M}$. Atiyah and J. Berndt [19].

Another work of Arnold in the same field ${ }^{21}$ unified the Petrovsky-Oleinik inequalities concerning topology of real hypersurfaces (or their complements) and brought mixed Hodge structures (just introduced by Steenbrink into complex singularity theory) into real algebraic geometry.

Arnold's interest in the 16th Hilbert problem, Part II, on the number of limit cycles of polynomial ODE systems on the plane has been an open-ended search for simplifying formulations. One such formulation $^{22}$ (about the maximal number of limit cycles born under a nonconservative perturbation of a Hamiltonian system and equivalent to the problem about the number of zeroes of Abelian integrals over a family of real algebraic ovals) generated extensive research. The results here include the general deep finiteness theorems of Khovansky and Varchenko, Arnold's conjecture about nonoscillatory behavior of the Abelian integrals, his geometrization of higher-dimensional Sturm

\footnotetext{
${ }^{19}$ The situation of ovals of real algebraic plane curves, the involutions of four-dimensional smooth manifolds, and the arithmetic of integral quadratic forms, Funct. Anal. Appl. 5 (1971), no. 3, 1-9.

${ }^{20}$ Details were published much later in The branched covering $C \mathrm{P}^{2} \rightarrow S^{4}$, hyperbolicity and projective topology, Sibirsk. Mat. Zh. 29 (1988), no. 5, 36-47.

${ }^{21}$ The index of a singular point of a vector field, the Petrovsky-Oleinik inequalities, and mixed Hodge structures, Funct. Anal. Appl. 12 (1978), no. 1, 1-14.

${ }^{22}$ V. I. Arnold, O. A. Oleinik, Topology of real algebraic varieties, Vestnik Moskov. Univ. Ser. I Mat. Mekh. (1979), no. 6, 7-17.
}

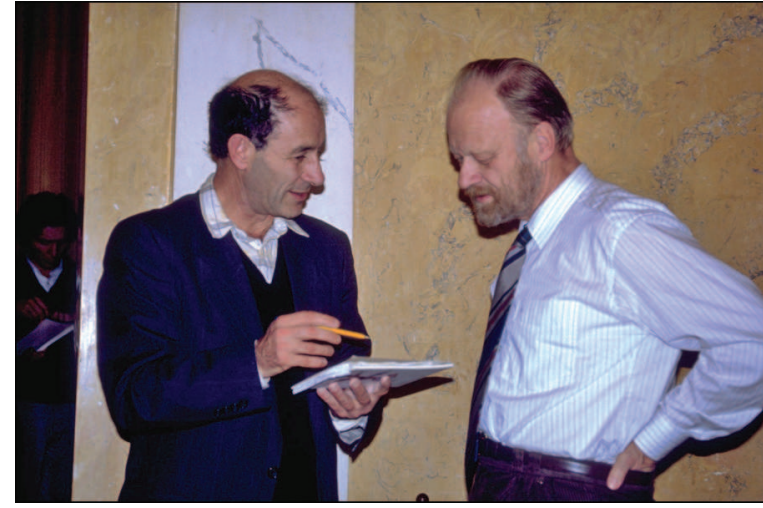

V. Arnold and J. Moser at the Euler Institute, St. Petersburg, 1991.

theory of (non)oscillations in linear Hamiltonian systems, ${ }^{23}$ various attempts to prove this conjecture (including a series of papers by Petrov-Tan'kin on Abelian integrals over elliptic curves, my own application of Sturm's theory to nonoscillation of hyperelliptic integrals, and more recent estimates of Grigoriev, Novikov-Yakovenko), and further work by Horozov, Khovansky, Ilyashenko and others. Yet another modification of the problem (a discrete one-dimensional analogue) suggested by Arnold led to a beautiful and nontrivial theorem of Yakobson in the theory of dynamical systems [39].

The classical problem in the theory of Diophantine approximations of inventing the higherdimensional analogue of continued fractions has been approached by many authors, with a paradoxical outcome: there are many relatively straightforward and relatively successful generalizations, but none as unique and satisfactory as the elementary continued fraction theory. Arnold's approach to this problem ${ }^{24}$ is based on his discovery of a relationship between graded algebras and Klein's sails (i.e., convex hulls of integer points inside simplicial convex cones in Euclidean spaces). Arnold's problems and conjectures on the subject have led to the results of E. Korkina and G. Lauchaud generalizing Lagrange's theorem (which identifies quadratic irrationalities with eventually periodic continued fractions) and to the work of Kontsevich-Sukhov generalizing Gauss's dynamical system and its ergodic properties. Thus the Klein-Arnold generalization, while not straightforward, appears to

\footnotetext{
${ }^{23}$ Sturm theorems and symplectic geometry, Funct. Anal. Appl. 19 (1985), no. 4, 1-10.

${ }^{24}$ A-graded algebras and continued fractions, Commun. Pure Appl. Math. 49 (1989), 993-1000; Higherdimensional continued fractions, Regul. Chaotic Dyn. 3 (1998), 10-17; and going back to Statistics of integral convex polyhedra, Funct. Anal. Appl. 14 (1980), no. 1, 1-3; and to the theory of Newton polyhedra.
} 
be just as unique and satisfactory as its classical prototype.

The above examples show how Arnold's interest in specific problems helped to transform them into central areas of modern research. There are other classical results which, according to Arnold's intuition, are scheduled to generate such new areas, but to my understanding have not yet achieved the status of important mathematical theories in spite of interesting work done by Arnold himself and some others. But who knows? To mention one: the Four-Vertex Theorem, according to Arnold, is the seed of a new (yet unknown) branch of topology (in the same sense as the Last Poincaré Theorem was the seed of symplectic topology). Another example: a field-theoretic analogue of Sturm theory, broadly understood as a study of topology of zero levels (and their complements) of eigenfunctions of selfadjoint linear partial differential operators.

Perhaps with the notable exceptions of KAMtheory and singularity theory, where Arnold's contributions are marked not only by fresh ideas but also by technical breakthroughs (e.g., a heavyduty tool in singularity theory-his spectral sequence), ${ }^{25}$ a more typical path for Arnold would be to invent a bold new problem, attack its first nontrivial cases with his bare hands, and then leave developing an advanced machinery to his followers. I have already mentioned how the theory of hyperplane arrangements emerged in this fashion. Here are some other examples of this sort where Arnold's work starts a new area.

In 1980 Arnold invented the concepts of Lagrangian and Legendrian cobordisms and studied them for curves using his theory of bifurcations of wave fronts and caustics. ${ }^{26}$ The general homotopy theory formulation was then given by Ya. Eliashberg, and the corresponding "Thom rings" computed in an award-winning treatise by M. Audin [20]. A geometric realization of Lagrange and Legendre characteristic numbers as the enumerative theory of singularities of global caustics and wave fronts was given by V. Vassiliev [38]. The method developed for this task, namely associating a spectral sequence to a stratification of functional spaces of maps according to types of singularities, was later applied by Vassiliev several more times, of which his work on Vassiliev invariants of knots is the most famous one.

Arnold's definition ${ }^{27}$ of the asymptotic Hopf invariant as the average self-linking number of

\footnotetext{
${ }^{25}$ A spectral sequence for the reduction of functions to normal forms, Funct. Anal. Appl. 9 (1975), 81-82.

${ }^{26}$ Lagrange and Legendre cobordisms. I, II, Funct. Anal. Appl. 14 (1980), no. 3, 1-13; no. 4, 8-17.

${ }^{27}$ See The asymptotic Hopf invariant and its applications, Selected translations. Selecta Math. Soviet. 5 (1986), no. 4, 327-345, which is the translation of a 1973 paper and one of Arnold's most frequently quoted works.
}

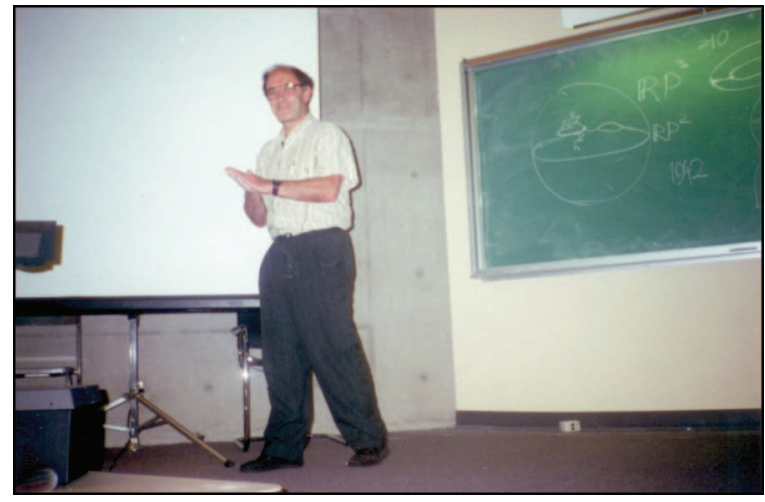

Lecturing in Toronto, 1997.

trajectories of a volume-preserving flow on a simply connected 3-fold and his "ergodic" theorem about coincidence of the invariant with Moffatt's helicity gave the start to many improvements, generalizations, and applications of topological methods in hydro- and magneto-dynamics due to M. H. Freedman et al., É. Ghys, B. Khesin, K. Moffatt, and many others. ${ }^{28}$

As one can find out, say, on MathSciNet, Arnold is one of the most prolific mathematicians of our time. His high productivity is partly due to his fearless curiosity and enormous appetite for new problems. ${ }^{29}$ Paired with his taste and intuition, these qualities often bring unexpected fruit, sometimes in the areas quite remote from the domain of his direct expertise. Here are some examples.

Arnold's observation ${ }^{30}$ on the pairs of triples of numbers computed by I. Dolgachev and A. Gabrielov and characterizing respectively uniformization and monodromy of 14 exceptional unimodal singularities of surfaces (in Arnold's classification) is known now under the name Arnold's Strange Duality. In 1977, due to Pinkham and Dolgachev-Nikulin, the phenomenon received a beautiful explanation in terms of geometry of K3-surfaces. As became clear in the early nineties, Arnold's Strange Duality was the first, and highly nontrivial, manifestation of Mirror Symmetry: a profound conjecture discovered by string theorists and claiming a sort of equivalence between symplectic topology and complex geometry (or singularity theory).

\footnotetext{
${ }^{28}$ See a review in Chapter III of V. I. Arnold, B. A. Khesin, Topological methods in hydrodynamics, Applied Math. Sciences, vol. 125, Springer-Verlag, NY, 1998.

${ }^{29}$ See the unusual book Arnold's Problems, SpringerVerlag, Berlin; PHASIS, Moscow, 2004.

${ }^{30}$ See Critical points of smooth functions, Proceedings of the International Congress of Mathematicians (Vancouver, B. C., 1974), Vol. 1, pp. 19-39.
} 
Arnold's work in pseudo-periodic geometry ${ }^{31}$ encouraged A. Zorich to begin a systematic study of dynamics on Riemann surfaces defined by levels of closed 1-forms, which led to a number of remarkable results of Kontsevich-Zorich [29] and others related to ergodic theory on Teichmüller spaces and conformal field theory, and of EskinOkounkov [24] in the Hurwitz problem of counting ramified covers over elliptic curves.

Arnold seems to be the first to suggest ${ }^{32}$ that monodromy (say of Milnor fibers or of flag varieties) can be realized by symplectomorphisms. The idea, picked up by M. Kontsevich and S. Donaldson, was upgraded to the monodromy action on the Fukaya category (consisting of all Lagrangian submanifolds in the fibers and of their Floer complexes). This construction is now an important ingredient of the Mirror Symmetry philosophy and gave rise to the remarkable results of $\mathrm{M}$. Khovanov and P. Seidel about faithfulness of such Hamiltonian representations of braid groups [27].

The celebrated Witten's conjecture proved by M. Kontsevich in 1991 characterizes intersection theory on Deligne-Mumford moduli spaces of Riemann surfaces in terms of KdV-hierarchy of integrable systems. A refreshingly new proof of this result was recently given by OkounkovPandharipande. A key ingredient in their argument is an elementary construction of Arnold from his work on enumerative geometry of trigonometric polynomials. ${ }^{33}$

Among many concepts owing Arnold their existence, let me mention two of general mathematical stature which do not carry his name.

One is the Maslov index, which proved to be important in geometry, calculus of variations, numbers theory, representation theory, quantization, index theory of differential operators, and whose topological origin was explained by Arnold. $^{34}$

The other one is the geometric notion of integrability in Hamiltonian systems. There is a lot of

\footnotetext{
${ }^{31}$ Topological and ergodic properties of closed 1-forms with incommensurable periods, Funct. Anal. Appl. 25 (1991), no. 2, 1-12.

${ }^{32}$ See Some remarks on symplectic monodromy of Milnor fibrations, The Floer memorial volume, 99-103, Progr. Math., 133, Birkhäuser, Basel, 1995.

${ }^{33}$ Topological classification of complex trigonometric polynomials and the combinatorics of graphs with an identical number of vertices and edges, Funct. Anal. Appl. 30 (1996), no. 1, 1-17.

${ }^{34}$ In his paper On a characteristic class entering into conditions of quantization, Funct. Anal. Appl. 1 (1967), $1-14$.
}

controversy over which of the known integrability mechanisms is most fundamental, but there is a consensus that integrability means a complete set of Poisson-commuting first integrals.

This definition and "Liouville's Theorem" on geometric consequences of the integrability property (namely, foliation of the phase space by Lagrangian tori) are in fact Arnold's original inventions.

Similar to the case with integrable systems, there are other examples of important developments which have become so common knowledge that Arnold's seminal role eventually became invisible. Let me round up these comments with a peculiar example of this sort.

The joint 1962 paper of Arnold and Sinai ${ }^{35}$ proves structural stability of hyperbolic linear diffeomorphisms of the 2-torus. Their idea, picked up by Anosov, was extended to his famous general stability theory of Anosov systems [2]. Yet, according to Arnold, the paper is rarely quoted, for the proof contained a mistake (although each author's contribution was correct, so that neither one could alone be held responsible). By the way, Arnold cites this episode as the reason why he refrained from writing joint research papers.

To reiterate what I said at the beginning, Vladimir Arnold has made outstanding contributions to many areas of pure mathematics and its applications, including those I described above and those I missed: classical and celestial mechanics, cosmology and hydrodynamics, dynamical systems and bifurcation theory, ordinary and partial differential equations, algebraic and geometric topology, number theory and combinatorics, real and complex algebraic geometry, symplectic and contact geometry and topology, and perhaps some others. I can think of few mathematicians whose work and personality would influence the scientific community at a comparable scale. And beyond this community, Arnold is a highly visible (and possibly controversial) figure, the subject of several interviews, of a recent documentary movie, and even of the night sky show, where one can watch an asteroid, Vladarnolda, named after him.

I am sure there are other mathematicians who also deserve [the name of the prize], but awarding it to Vladimir Arnold will hardly be perceived by anyone as a mistake.

\footnotetext{
${ }^{35}$ Arnold, V. I., Sinai, Ya. G., On small perturbations of the automorphisms of a torus, Dokl. Akad. Nauk SSSR 144 (1962), 695-698.
} 


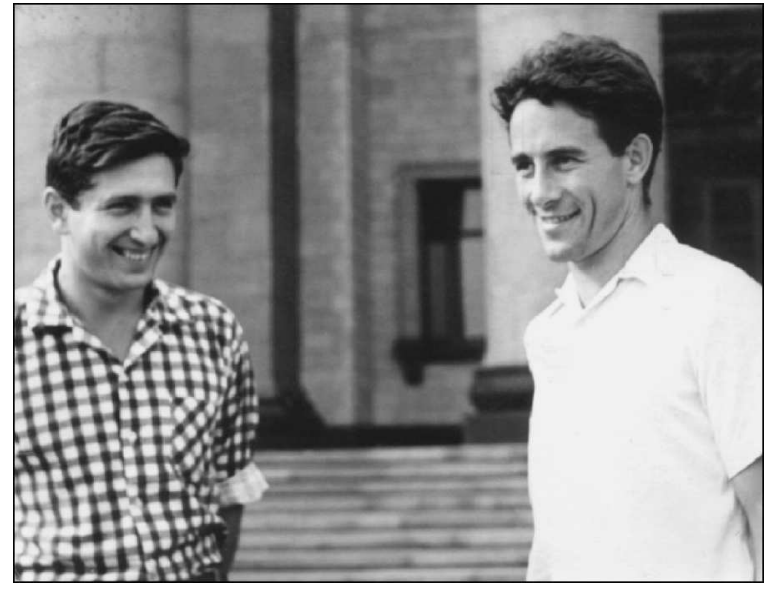

Ya. Sinai and V. Arnold, photo by J. Moser, 1963.

\section{Yakov Sinai}

\section{Remembering Vladimir Arnold: Early Years}

De mortuis veritas ${ }^{37}$

My grandparents and Arnold's grandparents were very close friends since the beginning of the twentieth century. Both families lived in Odessa, which was a big city in the southern part of Russia and now is a part of Ukraine. At that time, Odessa was a center of Jewish intellectual life, which produced many scientists, musicians, writers, and other significant figures.

My maternal grandfather, V. F. Kagan, was a well-known geometer who worked on the foundations of geometry. During World War I, he gave the very first lecture course in Russia on the special relativity theory. At various times his lectures were attended by future famous physicists L. I. Mandelshtam, I. E. Tamm, and N. D. Papaleksi. In the 1920s all these people moved to Moscow.

L. I. Mandelshtam was a brother of Arnold's maternal grandmother. He was the founder and the leader of a major school of theoretical physics that included A. A. Andronov, G. S. Landsberg, and M. A. Leontovich, among others. A. A. Andronov is known to the mathematical community for his famous paper "Robust systems", coauthored with L. S. Pontryagin, which laid the foundations of the theory of structural stability of dynamical systems. A. A. Andronov was the leader of a group of physicists and mathematicians working in Nizhny Novgorod, formerly Gorky, on nonlinear oscillations. M. A. Leontovich was one of the leading physicists in the Soviet Union. In the 1930s

Yakov Sinai is professor of mathematics at Princeton University. His email address is sinai@math. princeton . edu.

${ }^{37}$ About those who have died, only the truth. he coauthored with A. N. Kolmogorov the wellknown paper on the Wiener sausage. I. E. Tamm was a Nobel Prize winner in physics in the fifties. N. D. Papaleksi was a great expert on nonlinear optics.

V. I. Arnold was born in Odessa, where his mother had come for a brief visit with her family. She returned to Moscow soon after her son's birth. When Arnold was growing up, the news that his family had a young prodigy soon became widely known. In those days, when we were both in high school, we did not really know each other. On one occasion, Arnold visited my grandfather to borrow a mathematics book, but I was not there at the time. We met for the first time when we were both students at the mathematics department of the Moscow State University; he was walking by with Professor A. G. Vitushkin, who ran a freshman seminar on real analysis, and Arnold was one of the most active participants. When Arnold was a third-year undergraduate student, he was inspired by A. N. Kolmogorov to work on superposition of functions of several variables and the related Hilbert's thirteenth problem. Eventually this work became Arnold's Ph.D. thesis. When I visited the University of Cambridge recently, I was very pleased to learn that one of the main lecture courses there was dedicated to Arnold's and Kolmogorov's work on Hilbert's thirteenth problem.

Arnold had two younger siblings: a brother, Dmitry, and a sister, Katya, who was the youngest. The family lived in a small apartment in the center of Moscow. During one of my visits, I was shown a tent in the backyard of the building where Arnold used to spend his nights, even in cold weather. It seems likely that Arnold's excellent knowledge of history and geography of Moscow, which many of his friends remember with admiration, originated at that time.

Like me, Arnold loved nature and the outdoors. We did hiking and mountain climbing together. Since I knew Arnold so closely, I often observed that his ideas both in science and in life came to him as revelations. I remember one particular occasion, when we were climbing in the Caucasus Mountains and spent a night with some shepherds in their tent. In the morning we discovered that the shepherds were gone and had left us alone with their dogs. Caucasian dogs are very big, strong, and dangerous, for they are bred and trained to fight wolves. We were surrounded by fiercely barking dogs, and we did not know what to do. Then, all of a sudden, Arnold had an idea. He started shouting very loudly at the dogs, using all the obscenities he could think of. I never heard him use such language either before or after this incident, nor did anybody else. It was a brilliant idea, for it worked! The dogs did not touch Arnold and barely 


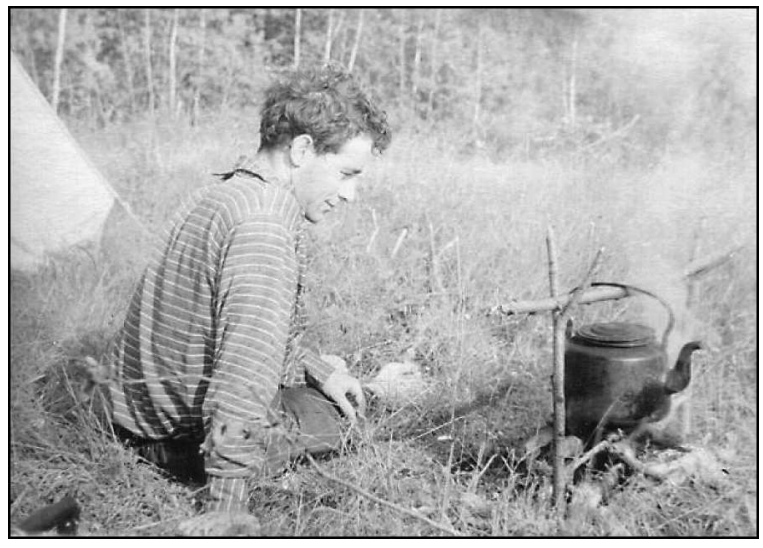

A hiking expedition, 1960s.

touched me. The shepherds returned shortly afterwards, and we were rescued.

On another occasion, roughly at the same time, as Anosov, Arnold, and I walked from the main Moscow University building to a subway station, which usually took about fifteen minutes, Arnold told us that he recently came up with the Galois theory entirely on his own and explained his approach to us. The next day, Arnold told us that he found a similar approach in the book by Felix Klein on the mathematics of the nineteenth century. Arnold was always very fond of this book, and he often recommended it to his students.

Other examples of Arnold's revelations include his discovery of the Arnold-Maslov cocycle in the theory of semi-classical approximations and Arnold inequalities for the number of ovals in real algebraic curves. Many other people who knew Arnold personally could provide more examples of this kind.

Arnold became a graduate student at the Moscow State University in 1959. Naturally it was A. N. Kolmogorov who became his advisor. In 1957 Kolmogorov gave his famous lecture course on dynamical systems, which played a pivotal role in the subsequent development of the theory. The course was given three years after Kolmogorov's famous talk at the Amsterdam Congress of Mathematics.

Kolmogorov began his lectures with the exposition of the von Neumann theory of dynamical systems with pure point spectrum. Everything was done in a pure probabilistic way. Later Kolmogorov found a similar approach in the book by Fortet and Blank-Lapierre on random processes, intended for engineers.

This part of Kolmogorov's lectures had a profound effect on researchers working on the measure-theoretic isomorphism in dynamical systems, a long-standing problem that goes back to von Neumann. It was shown that when the spectrum is a pure point one, it is the only isomorphism invariant of a dynamical system and that two systems with the same pure point spectrum are isomorphic. The excitement around these results was so profound that people began to believe that the isomorphism theory of systems with continuous spectrum would be just a straightforward generalization of the theory of systems with pure point spectrum. However, this was refuted by Kolmogorov himself. He proposed the notion of entropy as a new isomorphism invariant for systems with continuous spectrum. Since the entropy is zero for systems with pure point spectrum, it does not distinguish between such systems, but systems with continuous spectrum might have positive entropy that must be preserved by isomorphisms. This was a pathbreaking discovery, which had a tremendous impact on the subsequent development of the theory.

The second part of Kolmogorov's lectures was centered around his papers on the preservation of invariant tori in small perturbations of integrable Hamiltonian systems, which were published in the Doklady of the Soviet Academy of Sciences. Unfortunately there were no written notes of these lectures. V. M. Tikhomirov, one of Kolmogorov's students, hoped for many years to locate such notes, but he did not succeed. Arnold used to claim in his correspondence with many people that good mathematics students of Moscow University could reconstruct Kolmogorov's proof from the text of his papers in the Doklady. However, this was an exaggeration. Recently two Italian mathematicians, A. Giorgilli and L. Chierchia, produced a proof of Kolmogorov's theorem, which was complete and close to Kolmogorov's original proof, as they claimed.

Apparently Kolmogorov himself never wrote a detailed proof of his result. There might be several explanations. At some point, he had plans to work on applications of his technique to the famous three-body problem. He gave a talk on this topic at a meeting of the Moscow Mathematical Society. However, he did not prepare a written version of his talk. Another reason could be that Kolmogorov started to work on a different topic and did not want to be distracted. There might be a third reason, although some people would disagree with it. It is possible that Kolmogorov underestimated the significance of his papers. For example, some graduate exams on classical mechanics included the proof of Kolmogorov's theorem, so it was easy to assume that the proof was already known. The theory of entropy, introduced by Kolmogorov roughly at the same time, seemed a hotter and more exciting area. He might have felt compelled to turn his mind to this new topic. 


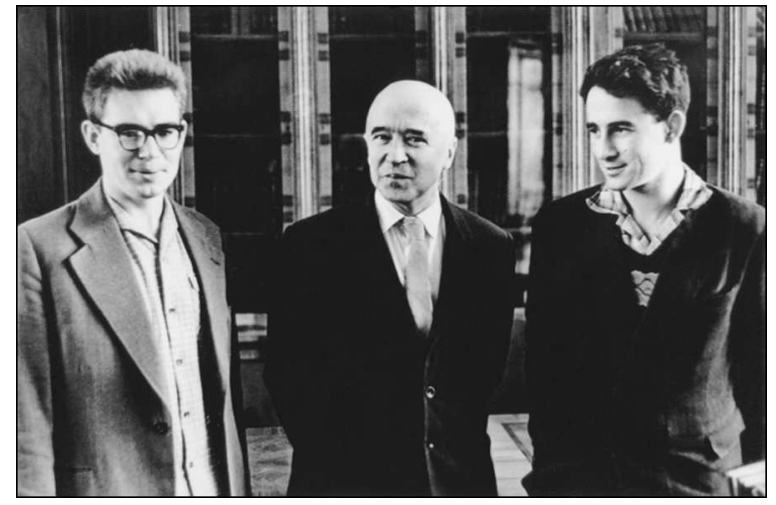

A. Kirillov, I. Petrovskii, V. Arnold.

Arnold immediately started to work on all the problems raised in Kolmogorov's lectures. In 1963 the Moscow Mathematical Society celebrated Kolmogorov's sixtieth birthday. The main meeting took place in the Ceremony Hall of the Moscow State University, with about one thousand people attending. The opening lecture was given by Arnold on what was later called KAM theory, where KAM stands for Kolmogorov, Arnold, Moser. For that occasion, Arnold prepared the first complete exposition of the Kolmogorov theorem. I asked Arnold why he did that, since Kolmogorov presented his proof in his lectures. Arnold replied that the proof of the fact that invariant tori constitute a set of positive measure was not complete. When Arnold asked Kolmogorov about some details of his proof, Kolmogorov replied that he was too busy at that time with other problems and that Arnold should provide the details by himself. This was exactly what Arnold did. I believe that when Kolmogorov prepared his papers for publication in the Doklady, he did have complete proofs, but later he might have forgotten some details. Perhaps it can be expressed better by saying that it required from him an effort that he was not prepared to make at that time.

In the following years, Kolmogorov ran a seminar on dynamical systems, with the participation of many mathematicians and physicists. At some point, two leading physicists, L. A. Arzimovich and M. A. Leontovich, gave a talk at the seminar on the existence of magnetic surfaces. Subsequently this problem was completely solved by Arnold, who submitted his paper to the main physics journal in the Soviet Union, called JETP. After some time, the paper was rejected. According to Arnold, the referee report said that the referee did not understand anything in that paper and hence nobody else would understand it. M. A. Leontovich helped Arnold to rewrite his paper in the form accessible to physicists, and it was published eventually. According to Arnold, this turned out to be one of his most quoted papers.
Arnold's first paper related to the KAM theory was about smooth diffeomorphisms of the circle that were close to rotations. Using the methods of the KAM theory, Arnold proved that such diffeomorphisms can be reduced to rotations by applying smooth changes of variables. The problem in the general case was called the Arnold problem. It was completely solved by M. Herman and J.-C. Yoccoz.

A. N. Kolmogorov proved his theorem in the KAM theory for the so-called nondegenerate perturbations of integrable Hamiltonian systems. Arnold extended this theorem to degenerate perturbations, which arise in many applications of the KAM theory.

Arnold proposed an example of the Hamiltonian system which exhibits a new kind of instability and which was later called the Arnold diffusion. The Arnold diffusion appears in many physical problems. New mathematical results on the Arnold diffusion were recently proved by J. Mather. V. Kaloshin found many applications of the Arnold diffusion to problems of celestial mechanics.

In later years Arnold returned to the theory of dynamical systems only occasionally. One can mention his results in fluid mechanics (see his joint book with B. Khesin [16]) and a series of papers on singularities in the distribution of masses in the universe, motivated by Y. B. Zeldovich. But all this was done in later years.

\section{Steve Smale}

\section{Vladimir I. Arnold}

My first meeting with V. I. Arnold took place in Moscow in September 1961 (certainly I had been very aware of him through Moser). After a conference in Kiev, where I had gotten to know Anosov, I visited Moscow, where Anosov introduced me to Arnold, Novikov, and Sinai. As I wrote later [35], I was extraordinarily impressed by such a powerful group of four young mathematicians and that there was nothing like that in the West. At my next visit to Moscow for the world mathematics congress in 1966 [36], I again saw much of Dima Arnold. At that meeting he introduced me to Kolmogorov.

Perhaps the last time I met Dima was in June 2003 at the one-hundred-year memorial conference for Kolmogorov, again in Moscow. In the intervening years we saw each other on a number of occasions in Moscow, in the West, and even in Asia.

Arnold was visiting Hong Kong at the invitation of Volodya Vladimirov for the duration of the

Stephen Smale is professor of mathematics at Toyota Technological Institute at Chicago and City University of Hong Kong. His email address is smale@cityu. edu.hk. 
fall semester of 1995, while we had just moved to Hong Kong. Dima and I often were together on the fantastic day hikes in the Hong Kong countryside parks. His physical stamina was quite impressive. At that time we two were also the focus of a wellattended panel on contemporary issues of mathematics at the Hong Kong University of Science and Technology. Dima expressed himself in his usual provocative way! I recall that we found ourselves on the same side in most of the controversies, and catastrophe theory in particular.

Dima Arnold was a great mathematician, and here I will just touch on his mathematical contributions which affected me the most.

While I never worked directly in the area of KAM, nevertheless those results had a great impact in my scientific work. For one thing they directed me away from trying to analyze the global orbit structures of Hamiltonian ordinary differential equations, in contrast to what I was doing for (unconstrained) equations. Thus KAM contributed to my motivation to study mechanics in 1970 from the point of view of topology, symmetry, and relative equilibria rather than its dynamical properties. The work of Arnold had already affected those subjects via his big paper on fluid mechanics and symmetry in 1966. See Jerry Marsden's account of how our two works are related [32]. I note that Jerry died even more recently than Dima.

KAM shattered the chain of hypotheses, ergodic, quasi-ergodic, and metric transitivity going from Boltzmann to Birkhoff. That suggested to me some kind of non-Hamiltonian substitute in these hypotheses in order to obtain foundations for thermodynamics [37].

I read Arnold's paper on braids and the cohomology of swallowtails. It was helpful in my work on topology and algorithms, which Victor Vassiliev drastically sharpened.

Dima could express important ideas simply and in such a way that these ideas could transcend a single discipline. His work was instrumental in transforming Kolmogorov's early sketches into a revolutionary recasting of Hamiltonian dynamics with sets of invariant curves, tori of positive measure, and Arnold diffusion.

It was my good fortune to have been a part of Dima Arnold's life and his mathematics.

\section{Mikhail Sevryuk}

\section{Some Recollections of Vladimir Igorevich}

A very large part of my life is connected with Vladimir Igorevich Arnold. I became his student in the beginning of 1980 when I was still a

Mikhail Sevryuk is a senior researcher at the Russian Academy of Sciences. His email address is sevryuk@mccme.ru.

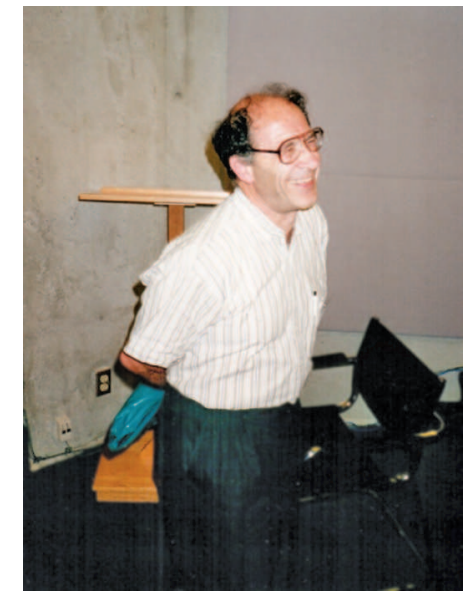

At Arnoldfest, Toronto, 1997.

freshman at the Department of Mechanics and Mathematics of Moscow State University. Under his supervision, I wrote my term papers, master's thesis, and doctoral thesis. At the end of my first year in graduate school, Arnold suggested that I write a monograph on reversible dynamical systems for Springer's Lecture Notes in Mathematics series, and working on this book was one of the cornerstones of my mathematical biography. For the last time, I met Vladimir Igorevich (V. I., for short) on November 3, 2009, at his seminar at Moscow State.

If I had to name one characteristic feature of Arnold as I remember him, I would choose his agility. He walked fast at walkways of Moscow State (faster than most of the students, not to mention the faculty), his speech was fast and clear, his reaction to one's remark in a conversation was almost always instantaneous, and often utterly unexpected. His fantastic scientific productivity is well known, and so is his enthusiasm for sports.

V. I. always devoted a surprising amount of time and effort to his students. From time to time, he had rather weak students, but I do not recall a single case when he rejected even a struggling student. In the 1980s almost every meeting of his famous seminar at Moscow State he started with "harvesting": collecting notes of his students with sketches of their recent mathematical achievements or drafts of their papers (and Arnold returned the previously collected ones with his corrections and suggestions). After a seminar or a lecture, he often continued talking with participants for another 2-3 hours. Arnold's generosity was abundant. Many times, he gave long written mathematical consultations, even to people unknown to him, or wrote paper reports substantially exceeding the submitted papers. 


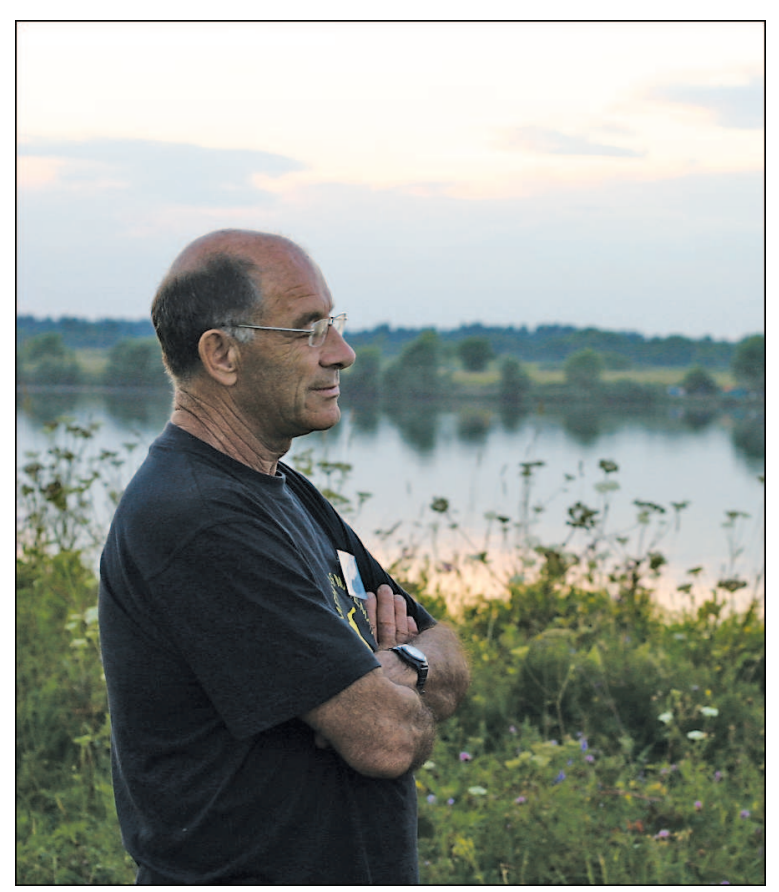

Dubna, 2006.

In recent years, he used all his energy to stop a rapid deterioration of mathematical (and not only mathematical) education in Russia.

I tried to describe my experience of being V. I.'s student at Moscow State in [34]. I would like to emphasize here that Arnold did not follow any pattern in supervising his students. In some cases he would inform a student that there was a certain "uninhabited" corner in the vast mathematical land, and if the student decided to "settle" at that corner, then it was this student's task to find the main literature on the subject, to study it, to pose new problems, to find methods of their solution, and to achieve all this practically single-handedly. Of course, V. I. kept the progress under control. (I recall that, as a senior, I failed to submit my "harvest" for a long time, but finally made substantial progress. Arnold exclaimed, "Thank God, I have started fearing that I would have to help you!") But in other situations, Arnold would actively discuss a problem with his student and invite him to collaborate - this is how our joint paper [13] came about. When need be, V. I. could be rather harsh. Once I witnessed him telling a student, "You are working too slowly. I think it will be good if you start giving me weekly reports on your progress." Arnold never tried to spare one's self-esteem.

V. I. had a surprising feeling of the unity of mathematics, of natural sciences, and of all nature. He considered mathematics as being part of physics, and his "economics" definition of mathematics as a part of physics in which experiments are cheap is often quoted.
(Let me add in parentheses that I would prefer to characterize mathematics as the natural science that studies the phenomenon of infinity by analogy with a little-known but remarkable definition of topology as the science that studies the phenomenon of continuity.) However, Arnold noted other specific features of mathematics: "It is a fair observation that physicists refer to the first author, whereas mathematicians to the latest one." (He considered adequate references to be of paramount importance and paid much attention to other priority questions; this was a natural extension of his generosity, and he encouraged his students to "over-acknowledge", rather than to "under-acknowledge".)

V. I. was an avid fighter against "Bourbakism", a suicidal tendency to present mathematics as a formal derivation of consequences from unmotivated axioms. According to Arnold, one needs mathematics to discover new laws of nature as opposed to "rigorously" justify obvious things. V. I. tried to teach his students this perception of mathematics and natural sciences as a unified tool for understanding the world. For a number of reasons, after having graduated from university, I had to work partially as a chemist, and after Arnold's school this caused me no psychological discomfort.

Fundamental mathematical achievements of Arnold, as well as those of his teacher, A. N. Kolmogorov, cover almost all mathematics. It well may be that V. I. was the last universal mathematician. My mathematical specialization is the KAM theory. V. I. himself described the contributions of the three founders; see, e.g., [15], [17]. For this reason, I shall only briefly recall Arnold's role in the development of the KAM theory.

KAM theory is the theory of quasiperiodic motions in nonintegrable dynamical systems. In 1954 Kolmogorov made one of the most astonishing discoveries in mathematics of the last century. Consider a completely integrable Hamiltonian system with $n$ degrees of freedom, and let $(I, \varphi)$ be the corresponding action-angle variables. The phase space of such a system is smoothly foliated into invariant $n$-tori $\{I=$ const $\}$ carrying conditionally periodic motions $\dot{\varphi}=\omega(I)$. Kolmogorov showed that if $\operatorname{det}(\partial \omega / \partial I) \neq 0$, then (in spite of the general opinion of the physical community of that time) most of these tori (in the Lebesgue sense) are not destroyed by a small Hamiltonian perturbation but only slightly deformed in the phase space. To be more precise, a torus $\left\{I=I^{0}\right\}$ persists under a perturbation whenever the frequencies $\omega_{1}\left(I^{0}\right), \ldots, \omega_{n}\left(I^{0}\right)$ are Diophantine (strongly incommensurable). The perturbed tori (later called Kolmogorov tori) carry quasiperiodic motions with the same frequencies. To prove this fundamental theorem, Kolmogorov proposed a new, powerful method of constructing an infinite sequence of canonical coordinate 


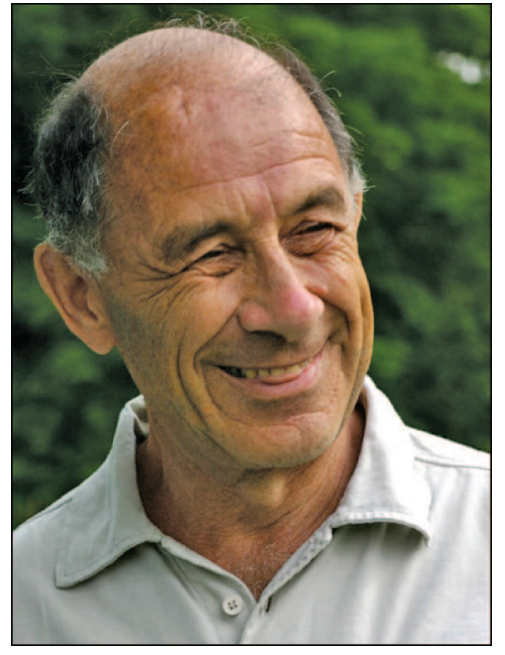

Vladimir Arnold.

transformations with accelerated ("quadratic") convergence.

Arnold used Kolmogorov's techniques to prove analyticity of the Denjoy homeomorphism conjugating an analytic diffeomorphism of a circle with a rotation (under the condition that this diffeomorphism is close to a rotation and possesses a Diophantine rotation number). His paper [4] with this result contained also the first detailed exposition of Kolmogorov's method. Then, in a series of papers, Arnold generalized Kolmogorov's theorem to various systems with degeneracies. In fact, he considered two types of degeneracies often encountered in mechanics and physics: the proper degeneracy, where some frequencies of the perturbed tori tend to zero as the perturbation magnitude vanishes, and the limit degeneracy, where the unperturbed foliation into invariant tori is singular and includes tori of smaller dimensions. The latter degeneracy is modeled by a one-degree-of-freedom Hamiltonian system having an equilibrium point surrounded by invariant circles (the energy levels). These studies culminated in Arnold's famous (and technically extremely hard) result [5] on stability in planetarylike systems of celestial mechanics where both the degeneracies combine.

Kolmogorov and Arnold dealt only with analytic Hamiltonian systems. On the other hand, J. K. Moser examined the finitely smooth case. The acronym "KAM" was coined by physicists F. M. Izrailev and B. V. Chirikov in 1968.

Arnold always regarded his discovery of the universal mechanism of instability of the action variables in nearly integrable Hamiltonian systems with more than two degrees of freedom [6] as his main achievement in the Hamiltonian perturbation theory. He also constructed an explicit example where such instability occurs. Chaotic evolution of the actions along resonances between the Kolmogorov tori was called "Arnold's diffusion" by Chirikov in 1969. In the case of two degrees of freedom, the Kolmogorov 2-tori divide a three-dimensional energy level, which makes an evolution of the action variables impossible.

All these works by Arnold took place in 19581965. At the beginning of the eighties, he returned to the problem of quasiperiodic motions for a short time and examined some interesting properties of the analogs of Kolmogorov tori in reversible systems. That was just the time when I started my diploma work. So V. I. forced me to grow fond of reversible systems and KAM theory, for which I'll be grateful to him forever.

I would like to touch on yet one more side of Arnold's research. In spite of what is occasionally claimed, Arnold did not hate computers: he considered them as an absolutely necessary instrument of mathematical modeling when indeed large computations were involved. He initiated many computer experiments in dynamical systems and number theory and sometimes participated in them (see [17]). But of course he strongly disapproved of the aggressive penetration of computer technologies into all pores of society and the tendency of a man to become a helpless and mindless attachment to artificial intelligence devices. One should be able to divide 111 by 3 without a calculator (and, better still, without scrap paper).

V. I. had a fine sense of humor. It is impossible to forget his somewhat mischievous smile. In conclusion, here are a couple of stories which might help to illustrate the unique charm of this person.

I remember how a speaker at Arnold's seminar kept repeating the words "one can lift" (a structure from the base to the total space of a bundle). Arnold reacted: "Looks like your talk is about results in weight-lifting."

On another occasion, Arnold was lecturing, and the proof of a theorem involved tedious computations: "Everyone must make these computations once-but only once. I made them in the past, so I won't repeat them now; they are left to the audience!"

In the fall of 1987 the Gorbachev perestroika was gaining steam. A speaker at the seminar was drawing a series of pictures depicting the perestroika (surgery) of a certain geometrical object as depending on a parameter. Arnold: "Something is not quite right here. Why is your central stratum always the same? Perestroika always starts at the center and then propagates to the periphery." 


\section{Askold Khovanskii and Alexander Varchenko}

\section{Arnold's Seminar, First Years}

In 1965-66, V. I. Arnold was a postdoc in Paris, lecturing on hydrodynamics and attending R. Thom's seminar on singularities. After returning to Moscow, Vladimir Igorevich started his seminar, meeting on Tuesdays from 4 to 6 p.m. It continued until his death on June 3 of 2010. We became Arnold's students in 1966 and 1968, respectively. The seminar was an essential part of our life. Among the first participants were R. Bogdanov, N. Brushlinskaya, I. Dolgachev, D. Fuchs, A. Gabrielov, S. Gusein-Zade, A. Kushnirenko, A. Leontovich, O. Lyashko, N. Nekhoroshev, V. Palamodov, A. Tyurin, G. Tyurina, V. Zakalyukin, and S. Zdravkovska.

V. I. Arnold had numerous interesting ideas, and to realize his plans he needed enthusiastic colleagues and collaborators. Every semester he started the seminar with a new list of problems and comments. Everyone wanted to be involved in this lively creative process. Many problems were solved, new theories were developed, and new mathematicians were emerging.

Here we will briefly describe some of the topics of the seminar in its first years, as well as the ski outings which were an integral part of the seminar.

\section{Hilbert's 13th Problem and Arrangements of Hyperplanes}

An algebraic function $x=x\left(a_{1}, \ldots, a_{k}\right)$ is a multivalued function defined by an equation of the form

$$
x^{n}+P_{1}\left(a_{1}, \ldots, a_{k}\right) x^{n-1}+\cdots+P_{n}\left(a_{1}, \ldots, a_{k}\right)=0
$$

where $P_{i}$ 's are rational functions.

Hilbert's 13th Problem: Show that the function $x(a, b, c)$, defined by the equation

$$
x^{7}+x^{3}+a x^{2}+b x+c=0,
$$

cannot be represented by superpositions of continuous functions in two variables.

A. N. Kolmogorov and V. I. Arnold proved that in fact such a representation does exist [3], thus solving the problem negatively. Despite this result it is still believed that the representation is impossible if one considers the superpositions of (branches of) algebraic functions only.

Can an algebraic function be represented as a composition of radicals and arithmetic operations? Such a representation does exist if and only

Askold Khovanskii is professor of mathematics at the University of Toronto. His email address is askold@math. toronto. edu.

Alexander Varchenko is professor of mathematics at the University of North Carolina at Chapel Hill. His email address is anv@emai1.unc.edu.

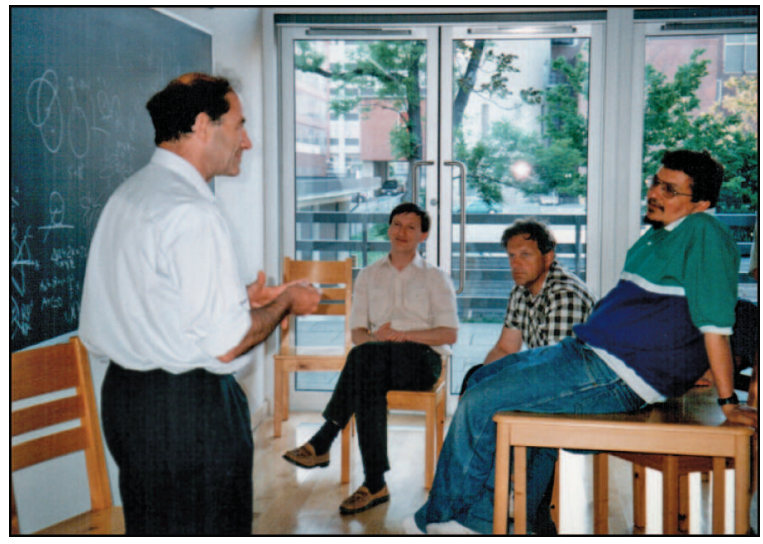

V. Arnold, Yu. Chekanov, V. Zakalyukin, and A. Khovanskii at Arnoldfest, Toronto, 1997.

if the Galois group of the equation over the field of its coefficients is solvable. Hence, the general algebraic function of degree $k \geq 5$, defined by the equation $a_{0} x^{k}+a_{1} x^{k-1}+\cdots+a_{k}=0$, cannot be represented by radicals.

In 1963, while teaching gifted high school students at Moscow boarding school No. 18, founded by Kolmogorov, V. I. Arnold discovered a topological proof of the insolvability by radicals of the general algebraic equation of degree $\geq 5$, a proof which does not rely on Galois theory. Arnold's lectures at the school were written down and published by V. B. Alekseev in [1].

V. I. Arnold often stressed that when establishing the insolvability of a mathematical problem, topological methods are the most powerful and those best suited to the task. Using such topological methods, V. I. Arnold proved the insolvability of a number of classical problems; see [18], [14]. Inspired by that approach, a topological Galois theory was developed later; see [28]. The topological Galois theory studies topological obstructions to the solvability of equations in finite terms. For example, it describes obstructions to the solvability of differential equations by quadratures.

The classical formula for the solution by radicals of the degree four equation does not define the roots of the equation only. It defines a 72valued algebraic function. V. I. Arnold introduced the notion of an exact representation of an algebraic function by superpositions of algebraic functions in which all branches of algebraic functions are taken into account. He proved that the algebraic function of degree $k=2^{n}$, defined by the equation $x^{k}+a_{1} x^{k-1}+\cdots+a_{k}=0$, does not have an exact representation by superpositions of algebraic functions in $<k-1$ variables; see [8] and the references therein. The proof is again topological and based on the characteristic classes of algebraic functions, introduced for that purpose. The characteristic classes are elements of 
the cohomology ring of the complement to the discriminant of an algebraic function. To prove that theorem V. I. Arnold calculated the cohomology ring of the pure braid group.

Consider the complement in $\mathbb{C}^{k}$ to the union of the diagonal hyperplanes,

$$
U=\left\{y \in \mathbb{C}^{k} \mid y_{i} \neq y_{j} \text { for all } i \neq j\right\} .
$$

The cohomology ring $H^{*}(U, \mathbb{Z})$ is the cohomology ring of the pure braid group on $k$ strings. The cohomology ring $H^{*}(U, \mathbb{Z})$ was described in [7]. Consider the ring $\mathcal{A}$ of differential forms on $U$ generated by the 1 -forms $w_{i j}=\frac{1}{2 \pi i} d \log \left(y_{i}-y_{j}\right), 1 \leq$ $i, j \leq k, i \neq j$. Then the relations $w_{i j}=w_{j i}$ and

$$
w_{i j} \wedge w_{j k}+w_{j k} \wedge w_{k i}+w_{k i} \wedge w_{i j}=0
$$

are the defining relations of $\mathcal{A}$. Moreover, the map $\mathcal{A} \rightarrow H^{*}(U, \mathbb{Z}), \alpha \mapsto[\alpha]$, is an isomorphism.

This statement says that each cohomology class in $H^{*}(U, \mathbb{Z})$ can be represented as an exterior polynomial in $w_{i j}$ with integer coefficients and the class is zero if and only if the polynomial is zero. As an application, V. I. Arnold calculated the Poincaré polynomial $P_{D}(t)=\sum_{i=0}^{k} \operatorname{rank} H^{i}(D) t^{i}$,

$$
P_{\Delta}(t)=(1+t)(1+2 t) \cdots(1+(n-1) t) .
$$

Arnold's paper [7] was the beginning of the modern theory of arrangements of hyperplanes; see, for example, the book by P. Orlik and H. Terao.

\section{Real Algebraic Geometry}

By Harnack's theorem, a real algebraic curve of degree $n$ in the real projective plane can consist of at most $g+1$ ovals, where $g=(n-1)(n-2) / 2$ is the genus of the curve. The M-curves are the curves for which this maximum is attained. For example, an M-curve of degree 6 has 11 ovals. Harnack proved that the M-curves exist.

If the curve is of even degree $n=2 k$, then each of its ovals has an interior (a disc) and an exterior (a Möbius strip). An oval is said to be positive if it lies inside an even number of other ovals and is said to be negative if it lies inside an odd number of other ovals. The ordinary circle, $x^{2}+y^{2}=1$, is an example of a positive oval.

In his 16th problem, Hilbert asked how to describe the relative positions of the ovals in the plane. In particular, Hilbert conjectured that 11 ovals on an M-curve of degree 6 cannot lie external to one another. This fact was proved by Petrovsky in 1938 [33].

The first M-curve of degree 6 was constructed by Harnack, the second by Hilbert. It was believed for a long time that there were no other M-curves of degree 6. Only in the 1960s did Gudkov construct a third example and prove that there are only three types of M-curves of degree 6; see [26].

Experimental data led Gudkov to the following conjecture: If $p$ and $m$ are the numbers of positive

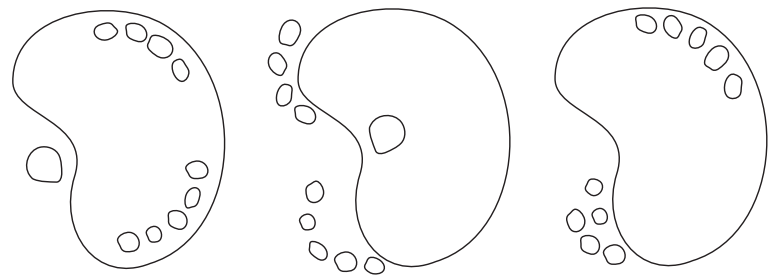

Harnack's, Hilbert's, and Gudkov's M-curves.

and negative ovals of an M-curve of degree $2 k$, then $p-m=k^{2} \bmod 8$.

V. I. Arnold was a member of Gudkov's Doctor of Science thesis defense committee and became interested in these problems. V. I. Arnold related Gudkov's conjecture and theorems of divisibility by 16 in the topology of oriented closed fourdimensional manifolds developed by V. Rokhlin and others. Starting with an M-curve, V. I. Arnold constructed a four-dimensional manifold with an involution and using the divisibility theorems proved that $p-m=k^{2} \bmod 4$; see [9]. Soon after that, V. A. Rokhlin, using Arnold's construction, proved Gudkov's conjecture in full generality.

This paper by V. I. Arnold began a revitalization of real algebraic geometry.

\section{Petrovsky-Oleinik Inequalities}

Petrovsky's paper [33] led to the discovery of remarkable estimates for the Euler characteristics of real algebraic sets, called Petrovsky-Oleinik inequalities. V. I. Arnold found in [10] unexpected generalizations of these inequalities and new proofs of the inequalities based on singularity theory.

Consider in $\mathbb{R}^{n+1}$ the differential one-form $\alpha=$ $P_{0} d x_{0}+P_{1} d x_{1}+\cdots+P_{n} d x_{n}$, whose components are homogeneous polynomials of degree $m$. What are possible values of the index ind of the form $\alpha$ at the point $0 \in \mathbb{R}^{n+1}$ ?

Let us introduce Petrovsky's number $\Pi(n, m)$ as the number of integral points in the intersection of the cube $0 \leq x_{0}, \ldots, x_{n} \leq m-1$ and the hyperplane $x_{0}+\cdots+x_{n}=(n+1)(m-1) / 2$. V. I. Arnold proved in [10] that

$$
\mid \text { ind } \mid \leq \Pi(n, m) \quad \text { and } \quad \text { ind } \equiv \Pi(n, m) \bmod 2 .
$$

His elegant proof of these relations is based on the Levin-Eisenbud-Khimshiashvili formula for the index of a singular point of a vector field.

Let $P$ be a homogeneous polynomial of degree $m+1$ in homogeneous coordinates on $\mathbb{R} P^{n}$. Petrovsky-Oleinik inequalities give upper bounds for the following quantities:

a) $|\chi(P=0)-1|$ for odd $n$, where $\chi(P=0)$ is the Euler characteristic of the hypersurface $P=0$ in $\mathbb{R} P^{n}$, and 
b) $|2 \chi(P \leq 0)-1|$ for even $n$ and $m+1$, where $\chi(P \leq 0)$ is the Euler characteristic of the subset $P \leq 0$ in $\mathbb{R} P^{n}$.

V. I. Arnold noticed in [10] that in both cases a) and b) the estimated quantity equals the absolute value of the index at $0 \in \mathbb{R}^{n+1}$ of the gradient of $P$. Thus, the Petrovsky-Oleinik inequalities are particular cases of Arnold's inequalities for $\alpha=$ $d P$.

Furthermore, Arnold's inequalities are exact (unlike the Petrovsky-Oleinik ones): for any integral value of ind with the properties $\mid$ ind $\mid \leq \Pi(n, m)$ and ind $\equiv \Pi(n, m) \bmod 2$ there exists a homogeneous 1 -form $\alpha$ (not necessarily exact) with this index (proved by Khovasnkii).

\section{Critical Points of Functions}

Critical points of functions was one of the main topics of the seminar in its first years. V. I. Arnold classified simple singularities of critical points in 1972, unimodal ones in 1973, and bimodal ones in 1975. Simple critical points form series $A_{n}, D_{n}, E_{6}, E_{7}, E_{8}$ in Arnold's classification. Already in his first papers V. I. Arnold indicated (sometimes without proofs) the connections of simple critical points with simple Lie algebras of the corresponding series. For example, the Dynkin diagram of the intersection form on vanishing cohomology at a simple singularity of an odd number of variables equals the Dynkin diagram of the corresponding Lie algebra, the monodromy group of the simple singularity equals the Weyl group of the Lie algebra, and the singularity index of the simple singularity equals $1 / N$, where $N$ is the Coxeter number of the Lie algebra.

One of the main problems of that time was to study characteristics of critical points. The methods were developed to calculate the intersection form on vanishing cohomology at a critical point (Gabrielov, Gusein-Zade), monodromy groups (Gabrielov, Gusein-Zade, Varchenko, Chmutov), and asymptotics of oscillatory integrals (Varchenko). The mixed Hodge structure on vanishing cohomology was introduced (Steenbrink, Varchenko), and the Hodge numbers of the mixed Hodge structure were calculated in terms of Newton polygons (Danilov, Khovanskii); see [12], [22] and the references therein.

The emergence of extensive new experimental data led to new discoveries. For example, according to Arnold's classification, the unimodal singularities form one infinite series $T_{p, q, r}$ and 14 exceptional families. Dolgachev discovered that the 14 exceptional unimodal singularities can be obtained from automorphic forms associated with the discrete groups of isometries of the Lobachevsky plane generated by reflections at the sides of some 14 triangles [23]. For the angles $\pi / p, \pi / q, \pi / r$ of such a triangle, the numbers $p, q, r$ are integers, called Dolgachev's triple. According to Gabrielov [25], the intersection form on vanishing cohomology at an exceptional unimodal singularity is described by another triple of integers, called Gabrielov's triple. V. I. Arnold noticed that Gabrielov's triple of an exceptional unimodal singularity equals Dolgachev's triple of (in general) another exceptional unimodal singularity, while Gabrielov's triple of that other singularity equals Dolgachev's triple of the initial singularity. Thus, there is an involution on the set of 14 exceptional unimodal singularities, called Arnold's strange duality. Much later, after discovery of the mirror symmetry phenomenon, it was realized that Arnold's strange duality is one of its first examples.

\section{Newton Polygons}

While classifying critical point of functions, Arnold noticed that, for all critical points of his classification, the Milnor number of the critical point can be expressed in terms of the Newton polygon of the Taylor series of that critical point. Moreover, an essential part of Arnold's classification was based on the choice of the coordinate system simplifying the Newton polygon of the corresponding Taylor series. (According to Arnold, he used "Newton's method of a moving ruler (line, plane)".) V. I. Arnold formulated a general principle: in the family of all critical points with the same Newton polygon, discrete characteristics of a typical critical point (the Milnor number, singularity index, Hodge numbers of vanishing cohomology, and so on) can be described in terms of the Newton polygon.

That statement was the beginning of the theory of Newton polygons. Newton polygons were one of the permanent topics of the seminar. The first result, the formula for the Milnor number in terms of the Newton polygon, was obtained by Kouchnirenko in [30]. After Kouchnirenko's report at Arnold's seminar, Lyashko formulated a conjecture that a similar statement must hold in the global situation: the number of solutions of a generic system of polynomial equations in $n$ variables with a given Newton polygon must be equal to the volume of the Newton polygon multiplied by $n$ !. Kouchnirenko himself proved this conjecture. David Bernstein [21] generalized the statement of Kouchnirenko's theorem to the case of polynomial equations with different Newton polygons and found a simple proof of his generalization. Khovanskii discovered the connection of Newton polygons with the theory of toric varieties and using this connection calculated numerous characteristics of local and global complete intersections in terms of Newton polygons; see [11] and the references therein. Varchenko calculated the zeta-function of the monodromy and 
asymptotics of oscillatory integrals in terms of Newton polygons; see [12].

Nowadays Newton polygons are a working tool in many fields. Newton polygons appear in real and complex analysis, representation theory, and real algebraic geometry; and the Newton polygons provide examples of mirror symmetry and so on.

\section{Skiing and Swimming}

Every year at the end of the winter Arnold's seminar went to ski on the outskirts of Moscow. This tradition started in 1973. While the number of seminar participants was between twenty and thirty people, no more than ten of the bravest participants came out to ski. People prepared for this event the whole winter. The meeting was at 8 a.m. at the railway station in Kuntsevo, the western part of Moscow, and skiing went on until after sunset, around 6 p.m. The daily distance was about $50 \mathrm{~km}$.

Usually Arnold ran in front of the chain of skiers, dressed only in swimming trunks. He ran at a speed a bit above the maximal possible speed of the slowest of the participants. As a result, the slowest participant became exhausted after an hour of such an outing and was sent back to Moscow on a bus at one of the crossroads. Then the entire process was repeated again and another participant was sent back to Moscow after another hour. Those who were able to finish the skiing were very proud of themselves.

Only one time was the skiing pattern different. In that year we were joined by Dmitri Borisovich Fuchs, a tall, unflappable man, who was at one time a serious mountain hiker. Early in the morning when Arnold started running away from the station with us, Dmitri Borisovich unhurriedly began to walk in the same direction. Soon he completely disappeared from our view, and Arnold stopped and began waiting impatiently for Fuchs to arrive. Arnold again rushed to run and Fuchs, again unperturbed, unhurriedly followed the group. So proceeded the entire day. That day none of the participants of the run were sent home in the middle of the day.

Several times we were joined by Olya Kravchenko and Nadya Shirokova, and every time they kept up the run as well as the best.

All participants of the ski-walk brought sandwiches, which they ate at a stop in the middle of the day. Before sandwiches there was bathing. In Moscow suburbs you will come across small rivers which are not frozen even in winter. We would meet at such a stream and bathe, lying on the bottom of the streambed as the water was usually only knee deep. We certainly did not use bathing suits, and there were no towels. The tradition of bathing in any open water at any time of the year Arnold had adopted from his teacher,

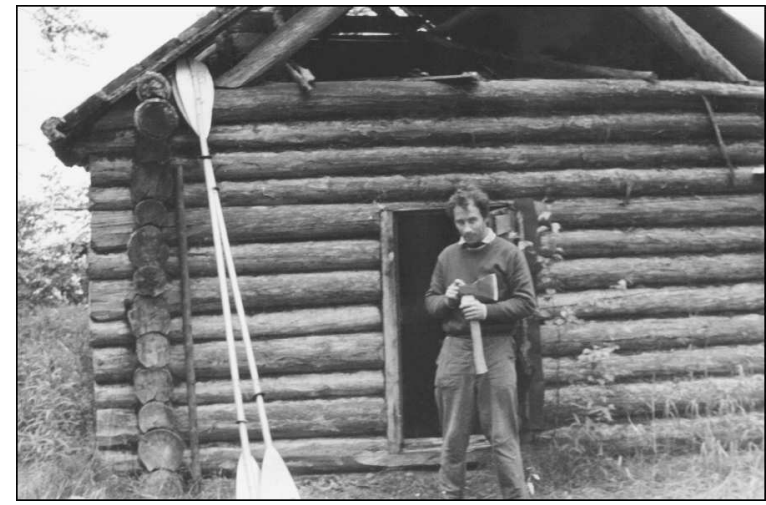

Summer expedition, 1960s.

Kolmogorov. This tradition was taken up by many participants of the seminar.

Arnold thought that vigorous occupation with mathematics should be accompanied by vigorous physical exercise. He skied regularly in the winter (about $100 \mathrm{~km}$ per week), and in summer rode a bicycle and took long walks.

There is a funny story connected to the tradition of bathing in any available open water. In 1983 the Moscow mathematicians were taken out to the Mathematical Congress in Warsaw. This congress had been boycotted by Western mathematicians. The large Soviet delegation was supposed to compensate for the small number of Western participants. A special Moscow-WarsawMoscow train had been arranged, which delivered us to Poland with Arnold. Once, walking across Warsaw in the evening with Arnold, we arrived at a bridge across the Vistula. While on the bridge we decided to bathe, as required by tradition. We reached the water in total darkness and swam for a few minutes. In the morning we found, to our amazement, that we were floating more in mud than water.

\section{Michael Berry}

\section{Memories of Vladimir Arnold}

My first interaction with Vladimir Arnold was receiving one of his notoriously caustic letters. In 1976 I had sent him my paper (about caustics, indeed) applying the classification of singularities of gradient maps to a variety of phenomena in optics and quantum mechanics. In my innocence, I had called the paper "Waves and Thom's theorem". His reply began bluntly:

Thank you for your paper. References:...

Michael Berry is professor of physics at the University of Bristol. His email address is Tracie.Anderson@bristo1.ac.uk. 
There followed a long list of his papers he thought I should have referred to. After declaring that in his view René Thom (whom he admired) never proved or even announced the theorems underlying his catastrophe theory, he continued:

I can't approve your system of referring to English translations where Russian papers exist. This has led to wrong attributions of results, the difference of 1 year being important-a translation delay is sometimes of 7 years...

and

...theorems and publications are very important in our science (... at present one considers as a publication rather 2-3 words at Bures or Fine Hall tea, than a paper with proofs in a Russian periodical)

and (in 1981)

I hope you'll not attribute these result [sic] to epigons.

He liked to quote Isaac Newton, often in scribbled marginal afterthoughts in his letters:

A man must either resolve to put out nothing new, or to become a slave to defend it

and (probably referring to Hooke)

Mathematicians that find out, settle and do all the business must content themselves with being nothing but dry calculators and drudges and another that does nothing but pretend and grasp at all things must carry away all the invention as well of those that were to follow him as of those that went before.

(I would not accuse Vladimir Arnold of comparing himself with Newton, but was flattered to be associated with Hooke, even by implication.)

I was not his only target. To my colleague John Nye, who had politely written "I have much admired your work...," he responded:

I understand well your letter, your admiration have not led neither to read the [reference to a paper] nor to send reprints....

This abrasive tone obviously reflected a tough and uncompromising character, but I was never offended by it. From the beginning, I recognized an underlying warm and generous personality, and this was confirmed when I finally met him in the late 1980s. His robust correspondence arose from what he regarded as systematic neglect by Western scientists of Russian papers in which their results had been anticipated. In this he was sometimes right and sometimes not. And he was unconvinced by my response that scientific papers can legitimately be cited to direct readers to the most accessible and readable source of a result rather than to recognize priority with the hard-to-find original publication.

He never lost his ironic edge. In Bristol, when asked his opinion of perestroika, he declared: "Maybe the fourth derivative is positive." And at a meeting in Paris in 1992, when I found, in my conference mailbox, a reprint on which he had written: "to Michael Berry, admiringly," I swelled with pride-until I noticed, a moment later, that every other participant's mailbox contained the same reprint, with its analogous dedication!

In 1999, when I wrote to him after his accident, he replied (I preserve his inimitable style):

...from the POINCARÉ hospital...the French doctors insisted that I shall recover for the following arguments: 1) Russians are 2 times stronger and any French would already die. 2) This particular person has a special optimism and 3) his humour sense is specially a positive thing: even unable to recognize you, he is laughing.... I do not believe this story, because it would imply a slaughtering of her husband for Elia, while I am still alive.

(Elia is Arnold's widow.)

There are mathematicians whose work has greatly influenced physics but whose writings are hard to understand; for example, I find Hamilton's papers unreadable. Not so with Arnold's: through his pellucid expositions, several generations of physicists came to appreciate the significance of pure mathematical notions that we previously regarded as irrelevant. "Arnold's cat" made us aware of the importance of mappings as models for dynamical chaos. And the exceptional tori that do not persist under perturbation (as Kolmogorov, Arnold, and Moser showed that most do) made us aware of Diophantine approximation in number theory: "resonant torus" to a physicist = "rational number" to a mathematician.

Most importantly, Arnold's writings were one of the two routes by which, in the 1970s, the notion of genericity slipped quietly into physics (the other route was critical phenomena in statistical mechanics, where it was called universality). Genericity emphasizes phenomena that are typical rather than the special cases (often with high symmetry) corresponding to exact solutions of the governing equations in terms of special functions. (And I distinguish genericity from abstract generality, which can often degenerate into what Michael Atiyah has called "general nonsense".) 
This resulted in a shift in our thinking whose significance cannot be overemphasized.

It suddenly occurs to me that in at least four respects Arnold was the mathematical counterpart of Richard Feynman. Like Feynman, Arnold made massive original contributions in his field, with enormous influence outside it; he was a master expositor, an inspiring teacher bringing new ideas to new and wide audiences; he was uncompromisingly direct and utterly honest; and he was a colorful character, bubbling with mischief, endlessly surprising.

\section{Acknowledgments}

The photographs are courtesy of the Arnold family archive, F. Aicardi, Ya. Eliashberg, E. Ferrand, B. and M. Khesin, J. Pöschel, M. Ratner, S. Tretyakova, and I. Zakharevich.

\section{References}

[1] V. B. Alekseev, Abel's Theorem in Problems and Solutions, based on the lectures of Professor V. I. Arnold, with a preface and an appendix by Arnold and an appendix by A. Khovanskii, Kluwer Academic Publishers, Dordrecht, 2004.

[2] D. ANosov, Geodesic flows on closed Riemannian manifolds of negative curvature, Trudy Mat. Inst. Steklov 90 (1967).

[3] V. I. ARNOLD, On functions of three variables, Dokl. Akad. Nauk SSSR 114 (1957), 679-681.

[4] V. ARNOLD, Small denominators. I. Mappings of a circle onto itself, Izvestiya AN SSSR, Ser. Mat. 25 (1961), 21-86.

[5] , Small denominators and problems of stability of motion in classical and celestial mechanics, Uspekhi Mat. Nauk 18 (1963), no. 6, 91-192.

[6] __ Instability of dynamical systems with many degrees of freedom, Dokl. Akad. Nauk SSSR 156 (1964), 9-12.

[7] braids, Mat. Zametki 5 (1969), 227-231.

[8] V. I. ARNOLD, The cohomology classes of algebraic functions that are preserved under Tschirnhausen transformations, Funkt. Anal. Prilozhen 4 (1970), no. $1,84-85$.

[9] V. ARNOLD, The situation of ovals of real algebraic plane curves, the involutions of four-dimensional smooth manifolds, and the arithmetic of integral quadratic forms, Funkt. Anal. Prilozhen 5 (1971), no. $3,1-9$.

[10] field, the Petrovsky-Oleinik inequalities, and mixed Hodge structures, Funct. Anal. Appl. 12 (1978), no. $1,1-14$.

[11] V. I. ARnold, A. B. Givental, A. G. KhovanSKII, A. N. VARCHENKO, Singularities of functions, wave fronts, caustics and multidimensional integrals, Mathematical Physics Reviews, Vol. 4, 1-92, Harwood Acad. Publ., Chur, 1984.

[12] V. ARnOLD, S. GUSEIN-ZADE, A. VARCHENKo, Singularities of Differentiable Maps, Vol. I. The Classification of Critical Points, Caustics and Wave Fronts, Birkhäuser, Boston, MA, 1985.
[13] V. ARnOld, M. SEVRyuk, Oscillations and bifurcations in reversible systems, in Nonlinear Phenomena in Plasma Physics and Hydrodynamics, Mir, Moscow, 1986, 31-64.

[14] V. I. ARNOLD, V. A. VASSILIEV, Newton's "Principia” read 300 years later, Notices Amer. Math. Soc. 36 (1989), no. 9, 1148-1154; 37 (1990), no. 2, 144.

[15] V. ARNOLD, From superpositions to KAM theory, in Vladimir Igorevich Arnold, Selected-60, PHASIS, Moscow, 1997, 727-740 (in Russian).

[16] V. ARNOLD, B. KHESIN, Topological Methods in Hydrodynamics, Springer-Verlag, New York, 1998.

[17] V. ARNOLD, From Hilbert's superposition problem to dynamical systems, in The Arnoldfest, Amer. Math. Soc., Providence, RI, 1999, 1-18.

[18] V. I. ARNOLD, I. G. PETROVSKII, Hilbert's topological problems, and modern mathematics, Russian Math. Surveys 57 (2002), no. 4, 833-845.

[19] M. ATIYAH, J. BeRndt, Projective planes, Severi varieties and spheres, Surveys in Differential Geometry, Vol. VIII (Boston, MA, 2002), 1-27, Int. Press, Somerville, MA, 2003.

[20] M. AUDIN, Cobordismes d'immersions lagrangiennes et legendriennes, Travaux en Cours, 20, Hermann, Paris, 1987.

[21] D. BERNSTEIN, On the number of roots of a system of equations, Funkt. Anal. i Prilozhen. 9 (1975), no. 3 , $1-4$.

[22] V. I. DANilov, A. G. KhovansKiI, Newton polyhedra and an algorithm for calculating Hodge-Deligne numbers, Math. USSR-Izv. 29 (1987), 279-298.

[23] I. DolgacheV, Conic quotient singularities of complex surfaces, Funkt. Anal. i Prilozhen. 8 (1974), no. $2,75-76$.

[24] A. Eskin, A. Okounkov, Asymptotics of numbers of branched coverings of a torus and volumes of moduli spaces of holomorphic differentials, Invent. Math. 145 (2001), 59-103.

[25] A. GABRIELOV, Dynkin diagrams of unimodal singularities, Funkt. Anal. i Prilozhen. 8 (1974), no. 3, $1-6$.

[26] D. GuDKov, Topology of real projective algebraic varieties, Uspekhi Mat. Nauk 29 (1974), no. 4, 3-79.

[27] M. Khovanov, P. SEIDEL, Quivers, Floer cohomology, and braid group actions, J. Amer. Math. Soc. 15 (2002), 203-271.

[28] A. G. KhOVANSKII, Topological Galois Theory, MTSNMO, Moscow, 2008.

[29] M. KONTSEVICH, A. ZORICH, Connected components of the moduli spaces of Abelian differentials with prescribed singularities, Invent. Math. 153 (2003), 631-678.

[30] A. Kouchnirenko, Polyèdres de Newton et nombres de Milnor, Invent. Math. 32 (1976), 1-31.

[31] N. KuIPER, The quotient space of $\mathbf{C} P^{2}$ by complex conjugation is the 4-sphere, Math. Ann. 208 (1974), 175-177.

[32] J. MARSDEN, Steve Smale and Geometric Mechanics, The Collected Papers of Stephen Smale, vol. 2, 871888, World Scientific Publ., River Edge, NJ, 2000.

[33] I. G. PetrovskiI, On the topology of real plane algebraic curves, Ann. of Math. (2) 39 (1938), 187-209.

[34] M. SEVRYuK, My scientific advisor V. I. Arnold, Matem. Prosveshchenie, Ser. 32 (1998), 13-18 (in Russian). 
[35] S. Smale, On how I got started in dynamical systems, 1959-1962, From Topology to Computation: Proceedings of the Smalefest, 22-26, Springer, New York, 1993.

[36] _ On the steps of Moscow University, From Topology to Computation: Proceedings of the Smalefest, 41-52, Springer, New York, 1993.

[37] _ On the problem of revising the ergodic hypothesis of Boltzmann and Birkhoff, The Collected Papers of Stephen Smale, vol. 2, 823-830, World Scientific Publ., River Edge, NJ, 2000.

[38] V. VASSILIEV, Lagrange and Legendre Characteristic Classes, Gordon and Breach Science Publ., New York, 1988.

[39] M. V. YAKOBSON, The number of periodic trajectories for analytic diffeomorphisms of a circle, Funct. Anal. Appl. 19 (1985), no. 1, 91-92.

\section{About the Cover \\ A mathematician's dinner table conversation}

It is often said that mathematicians are eager to draw on napkins, but in our experience it is actually rather rare. The doodles on the cover are by the late mathematician Vladimir Arnold, the subject of articles in this and the next issue of the Notices. Emmanuel Ferrand saved the napkin and took the photograph. He writes,

"Arnold wrote on this napkin at the occasion of a private meal at the Institut des Hautes Études Scientifiques near Paris. As far as I remember, it was early in 2006, and this is consistent with the problems discussed on this napkin. Most of what is written here is related to the question of the enumeration of the topological types of Morse functions on surfaces. The two drawings with the letters $A, B, C$ correspond to two of his favorite examples, the height functions of Stromboli and Etna, two famous volcanos in Italy. These are specifically referred to in Arnold's papers on the classification of Morse functions, and some of the problems he posed have been solved-some, for example, by Liviu Nicolaescu.

"It is important to note that Arnold's problems are one of his major contributions to mathematics. He wrote a list of about twenty open problems every year. He was not interested in leaving problems for the next generation like the Poincaré conjecture or the Riemann Hypothesis. On the contrary, the 'half life' of his problems was about five years. He also insisted not to take his questions too literally, but to consider them as a loose direction of research instead. He was not that much interested in questions whose answer is only 'yes' or 'no'. His seminars in Moscow and in Paris were structured around this problem list. Most of his problems were collected in the book Arnold's Problems from Springer-Verlag. There are still many interesting open questions waiting for the curious mathematician here!"

As anyone familiar with Arnold's writings knows, his line drawings, although not exactly requiring high technology to produce, were a charming and important part of his exposition. As the article in the next issue of the Notices points out, he insisted that figures play an important role in all of his students' work, as well.

- Bill Casselman Graphics editor

(notices-covers@ams.org) 